Mituro Hattori Junior

\title{
Fatores prognósticos em carcinomas de glândulas salivares recidivados submetidos à cirurgia de resgate
}

\begin{abstract}
Dissertação apresentada à Faculdade de Medicina da Universidade de São Paulo para obtenção do título de mestre em ciências
\end{abstract}

Área de concentração: Oncologia Orientador: Prof. Dr. Luiz Paulo Kowalski

São Paulo 


\section{Dedicatória}

A meus pais, pelo amor e dedicação em todas as fases da minha vida. A eles devo toda minha formação ética e moral.

Ao meu filho Gustavo, razão de tudo isso. 


\section{Agradecimentos}

Ao Prof. Dr. Luiz Paulo Kowalski, pela inestimável orientação, apoio e compreensão em todas as fases deste trabalho. Em especial agradeço pelo exemplo de pesquisador, médico e ser humano. Um exemplo que deve ser seguido por esta geração e as seguintes.

À Inês Nobuko Nishimoto, pelo estímulo, apoio e ajuda nos momentos complicados.

Ao Dr. Mauro Kasuo Ikeda, pelo estímulo, apoio e orientação em muitos momentos difíceis e decisivos.

Ao Dr. José Magrin, por todos os exemplos durante a residência de cirurgia oncológica no Hospital A.C. Camargo.

Ao Dr. André Lopes Carvalho, pelo estímulo e orientação.

À equipe da biblioteca do Hospital A.C. Camargo, em especial à Sra. Sueli Francisco. Sua ajuda foi fundamental para a formatação da dissertação e organização das referências bibliográficas.

À Srta. Elizângela Nivaldo Dias, por toda atenção e estímulo durante todo este período, orientando-me sobre as regras da pós-graduação.

À Sra. Raimunda Pereira, pelo auxílio na elaboração do banco de dados e também pelo carinho e amizade. 
Aos colegas do Departamento de Cirurgia de Cabeça e Pescoço do Hospital Anchieta: Dra. Jossi Ledo Kanda, Drs. Fábio Capelli, Renato Capuzzo e Fábio Pinto, pela compreensão, apoio e incentivo.

Aos Drs. Roberto Crisppi e Edicléia Morena, pela compreensão e apoio. 


\section{SUMÁRIO}

Lista de figuras

Lista de tabelas

Lista de abreviaturas

Resumo

Summary

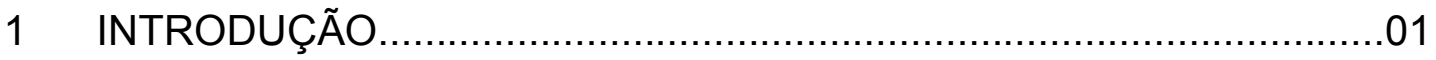

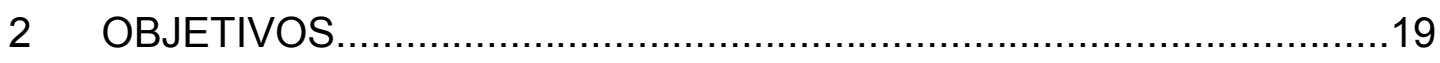

3 Casuística e métodos.................................................................21

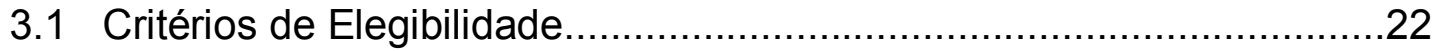

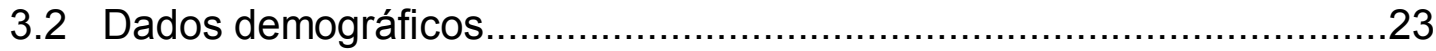

3.3 Dados relativos ao tumor primário e ao tipo histológico......................23

3.4 Dados relativos ao tratamento prévio e às recorrências......................25

3.5 Complicações e Óbitos............................................................... 32

3.6 Análise Estatística................................................................33

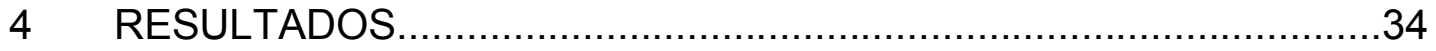

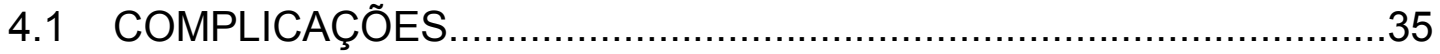

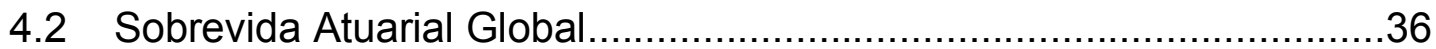

4.3 Recorrência após a cirurgia de resgate.........................................37

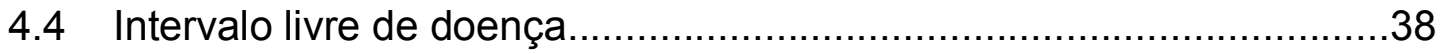

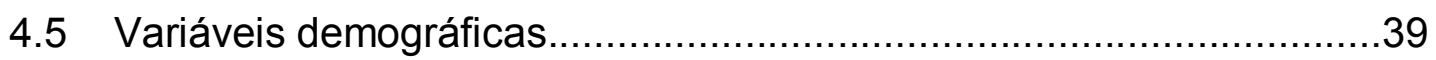

4.5.1 Faixa etária.......................................................................... 39

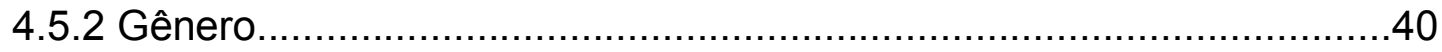

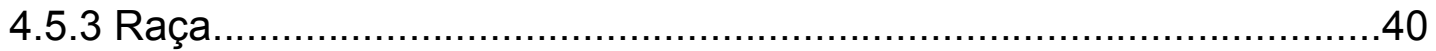

4.6 Variáveis clínicas..................................................................41

4.6.1 Localização do tumor primário.......................................................4 41

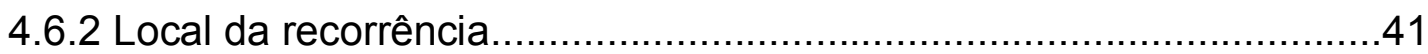

4.6.3 Comprometimento ósseo e de pele..........................................41

4.6.4 Estadiamento clínico da recidiva..............................................42

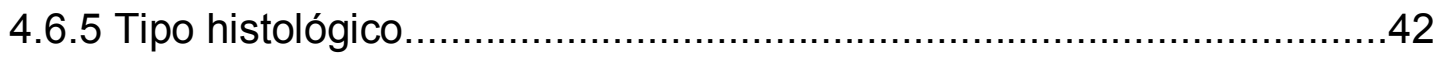




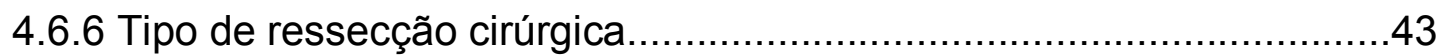

4.6.7 Conservação do nervo facial......................................................43

4.6.8 Radioterapia adjuvante após a cirurgia de resgate.........................44

5 DISCUSSÃO........................................................................... 46

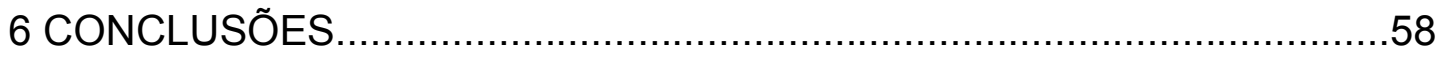

7 ANEXO

8 REFERÊNCIAS BIBLIOGRÁFICAS ..............................................66 


\section{LISTA DE FIGURAS}

Figura 1 Distribuição dos casos conforme a localização do tumor primário.

Figura 2 Distribuição dos casos de acordo com tipo da recorrência..........26

Figura 3 Sítio de recorrência conforme tratamento prévio......................26

Figura 4 Distribuição dos sítios de recorrência dos pacientes com tumores da parótida.

Figura 5 Distribuição dos sítios das recorrências em pacientes

com tumores da glândula submandibular.

Figura 6 Distribuição dos sítios de recorrência de tumores

de glândulas salivares menores intra orais

Figura 7 Distribuição dos casos segundo o estádio clínico da recorrência tumoral (rTNM).

Figura 8 Distribuição do tempo entre o tratamento inicial e a recorrência, em períodos (meses)....

Figura 9 Tipo de reconstrução na cirurgia de resgate.

Figura 10 Curva de sobrevida atuarial global dos 59 pacientes

submetidos à cirurgia de resgate por carcinomas

recidivados de glândulas salivares. 36

Figura 11 Curva de sobrevida atuarial de acordo com o tempo entre o tratamento inicial e a recorrência.

Figura 12 Curva de sobrevida atuarial global de acordo com

a faixa etária. 40 


\section{LISTA DE TABELAS}

Tabela 1 Distribuição dos casos de acordo com o tipo histológico e o local do tumor primário

Tabela 2 Tratamento cirúrgico da recorrência local, regional ou locorregional. 31

Tabela 3 Tipo de complicações locais após cirurgia de resgate para tumor de glândulas salivares.

Tabela 4 Distribuição da segunda recorrência de acordo com o sitio da primeira recorrência. 38

Tabela 5 Sobrevida atuarial global após cirurgia de resgate de acordo com variáveis clínicas e anatomopatológicas .44

Tabela 6 Sobrevida atuarial global após cirurgia de resgate de acordo com variáveis anatomopatológicas

e terapêuticas. 


\section{LISTA DE ABREVIATURA E SÍMBOLOS}

$\begin{array}{ll}\text { rEC } & \text { Estádio clínico da recidiva } \\ \mathrm{n} & \text { número de casos } \\ < & \text { menor que } \\ > & \text { maior que } \\ \text { RT } & \text { Radioterapia } \\ / & \text { por } \\ \% & \text { porcentagem } \\ \mathrm{p} & \text { valor estatísticos de } \mathrm{p} \\ \text { AJCC } & \text { American Joint Comitte on Cancer }\end{array}$




\section{RESUMO}

Hattori Junior M. Fatores prognósticos em carcinoma de glândulas salivares recidivados submetidos à cirurgia de resgate. [Dissertação]. São Paulo: Faculdade de Medicina, Universidade de São Paulo; 2007.

Carcinomas de glândulas salivares são raros e seu tratamento de escolha é cirúrgico, seguido ou não de radioterapia. Até $50 \%$ dos casos apresentam recorrências neoplásicas e a maioria delas é local. $\mathrm{O}$ único tratamento de resgate com potencial curativo é o cirúrgico. No entanto, poucos estudos abordam o tema. O objetivo deste estudo é avaliar a ocorrência de complicações cirúrgicas e os fatores prognósticos clínicos e anatomopatológicos em pacientes portadores de carcinoma de glândulas salivares recidivado, submetidos à cirurgia de resgate. Foram avaliados 59 pacientes que preenchiam os critérios de elegibilidade do estudo: diagnóstico comprovado da recidiva de carcinoma de glândulas salivares e cirurgia de resgate realizada com intenção curativa. Trinta e 5 pacientes desta série $(59,3 \%)$ foram tratados previamente em outra instituição. A cirurgia de resgate de todos os pacientes foi realizada no Hospital A. C. Camargo, em São Paulo, entre os anos de 1957 a 2000. O local do tumor primário foi parótida em 34 casos (57,6\%); cavidade oral, em $13(22,1 \%)$ e glândula submandibular, em 12 (20,3\%). O tipo histológico mais freqüente foi o carcinoma adenocístico em 23 casos (39\%), seguido pelo carcinoma mucoepidermóide, em 14 (23,7\%). O tratamento prévio foi somente cirurgia em 47 casos, cirurgia e radioterapia adjuvante em 11 casos e cirurgia e quimioterapia em apenas um caso. A maioria dos pacientes apresentou recorrência local $(39 ; 66 \%)$, em sete casos a recorrência foi regional $(11,9 \%)$ e em treze foi locorregional $(22,1 \%)$. O estádio clínico da recorrência foi inicial (rEC I e II) em 15 casos (25,5\%) e avançado (rEC III e IV), em 44 $(74,5 \%)$. O tratamento cirúrgico da recorrência foi ressecção local em 39 casos $(66,1 \%)$, esvaziamento cervical isolado em $7(11,9 \%)$ e cirurgias em 
monobloco (ressecção local associada a esvaziamento cervical) em 13 casos (22\%). Em 5 pacientes foi realizada parotidectomia ampliada com temporalectomia. A taxa global de complicações cirúrgicas foi de $32,2 \%$. A complicação mais comum foi a infecção da ferida operatória. Não houve mortalidade pós-operatória. Houve nova recorrência em 36 pacientes (61\%). A recorrência mais freqüente foi a distância, em 16 casos (27\%), sendo o pulmão o sítio mais acometido. Em 10 casos (17\%) houve falha no controle local; em 5 (8,5\%), falha locorregional e em 5 , falha regional (8,5\%). A sobrevida atuarial global, após a cirurgia de resgate, foi de $61,3 \%$ em 5 anos e $42 \%$ em 10 anos. O sexo, o tipo histológico, a topografia, o estadiamento clínico e a conservação do nervo facial (tumores parotídeos) não apresentaram associação com a sobrevida global. Em análise univariada a idade maior que 60 anos foi associada a pior prognóstico. Este estudo demonstra que o prognóstico de pacientes com carcinomas de glândulas salivares recidivados, selecionados para serem submetidos à cirurgia de resgate é aceitável. As taxas de complicações cirúrgicas foram geralmente locais e não houve mortalidade pós-operatória nesta série. 


\section{SUMMARY}

Hattori Junior M. Prognostic factors in salvage surgery for recurrent salivary gland carcinoma. [Dissertation]. São Paulo: Faculdade de Medicina, Universidade de São Paulo; 2007.

Salivary gland carcinomas are rare and the main treatment option is surgery with or without postoperative radiotherapy. Almost half of the cases present recurrences of tumors and the majority are local. The only potentially curative treatment for recurrent tumors is salvage surgery. However, few papers were published on this subject. The aim of this study is evaluate the surgical complications and clinical and pathologic prognostic factors in patients with recurrent salivary gland carcinoma submitted to salvage surgery. We reviewed the medical charts of 59 patients that fulfilled the eligibility critera of the study: proven diagnosis of locorregionally recurrent salivary gland carcinoma and surgery with curative intention. Thirty five patients $(59,3 \%)$ had been previously treated at another institution. The salvage surgery in all the patients was performed at the A. C. Camargo Hospital in São Paulo, Brazil from 1957 to 2000 . The site of the primary tumor was the parotid in 34 cases, the oral cavity in 13 and submandibular gland in 12 cases. The most frequent histopathologic type was adenoid cystic carcinoma (23 cases; $39 \%$ ) and mucoepidermoid carcinoma (14 cases $; 23.7 \%$ ). The prior treatment was surgery alone in 47 cases, surgery with adjuvant radiotherapy in 11 cases and surgery and adjuvant chemotherapy in only one case. The majority of the patients presented local recurrence (39; 66\%), in seven cases the recurrence was regional $(11.9 \%)$ and in thirteen cases it was locorregional $(22.1 \%)$. The clinical stage of the recurrences were initial (rCS I/II) in 15 patients $(25.5 \%)$ and advanced (rCS III/IV) in 44 patients (74.5\%). The surgical treatment of the recurrence was local ressection in 39 cases $(66.1 \%)$, en bloc surgeries (local ressection and neck dissection) in thirteen cases (22\%) and neck dissection alone in seven cases (11.9\%). Wide parotidectomy with temporal bone ressection was performed in five cases. The overall rate of surgical complications was $32.2 \%$. The most frequent complication was wound 
infection. There was no post-operative deaths. There was a new recurrence in $61 \%$ of the patients, and the majority were distant metastases (16 cases; $27 \%$ ). The lung was the most frequent involved site. In ten cases $(17 \%)$ the recurrence was local, in five cases it was locorregional (8.5\%). The actuarial overall survival were $61.3 \%$ in 5 years and $42 \%$ in ten years and in five cases it was regional (8.5\%). Gender, histopathology, site of the primary tumor, facial nerve preservation (parotid tumors), were not associated with overall survival. In the univariate analysis, only age older than 60 years was associate with a poor prognosis. This study shows that the prognosis of patients with recurrent salivary gland carcinoma treated to salvage surgery is acceptable. The rates of surgical complications are local and there was no post-operative mortality in this series. 


\section{INTRODUÇÃO}

Os tumores malignos de glândulas salivares compreendem um grupo heterogêneo de neoplasias quanto à apresentação clínica, histologia, comportamento biológico e prognóstico. Sua incidência representa 3 a $6 \%$ dos diagnósticos de câncer de cabeça e pescoço e 0,3\% de todos os cânceres (Spiro 1986, Spiro et al. 1989). Nos Estados Unidos a incidência é de 1/100.000 habitantes; no Brasil 0,9/100.000 (Parkin et al. 1997). Quanto à topografia, esses tumores podem se localizar em glândulas salivares maiores (parótidas, submandibulares e sublinguais) ou em glândulas salivares menores, situadas na submucosa do trato aerodigestivo superior. A maioria dos casos ocorre na parótida (70\%), seguida da glândula submandibular (8 a 10\%) (Spiro et al. 1989). A distribuição por sexo é igualitária, havendo em alguns trabalhos, predominância no sexo feminino (Wahlberg et al. 2002, Satko et al. 2000) e, em outros, no masculinos (Fitzpatrick et al. 1986, Lopes et al. 1998). Acometem crianças e adultos, porém com maior freqüência após a quinta década de vida (Speight et al. 2002). Os tumores de parótida em indivíduos adultos geralmente são benignos, enquanto em crianças há maior proporção de tumores malignos (mais de $50 \%$ dos casos) (Satko et al. 2000).

A classificação histológica dos tumores malignos de glândulas salivares é complexa, devido à diversidade de tipos encontrados, acarretando dificuldades para o diagnóstico e planejamento terapêutico. Algumas vezes somente é possível classificar esses tumores com base no exame da peça cirúrgica, inclusive com a utilização de painéis de 
marcadores por imunoistoquímica. A classificação proposta pela Organização Mundial de Saúde de 1972 (Trackray e Sobin 1972) divide os tipos histológicos em: carcinoma adenocístico, carcinoma mucoepidermóide, adenocarcinomas, carcinoma de células acinares, carcinoma epidermóide e carcinoma indiferenciado. Reviu-se esta nomenclatura em 1992 (Seifert et al. 1992), subdividindo-se os adenocarcinomas (polimórfico de baixo grau, de células basais, mucinoso) e acrescentando-se carcinoma sebáceo, cistadenocarcinoma papilífero, carcinoma oncocitico, carcinoma de ducto salivar, carcinoma epitelial-mioepitelial (mioepitelioma maligno), carcinoma ex-adenoma pleomórfico, carcinoma de pequenas células, carcinoma indiferenciado e outros. Para carcinomas mucoepidermóides, adenocarcinomas e carcinoma epidermóide empregam-se uma classificação segundo o grau histológico (baixo, intermediário e alto) (Spiro et al. 1989, da Cruz 2004).

Os tipos histológicos mais freqüentes são: carcinoma mucoepidermóide, carcinoma adenocístico e adenocarcinoma. Na literatura há controvérsia quanto ao tipo histológico mais coumum, mas a maioria dos trabalhos reporta o carcinoma mucoepidermóide (Spiro et al. 1989, Jasisyanont et al. 2002, Wahlberg et al. 2002). Entretanto, outros mostram maior incidência do carcinoma adenocístico (Kokemueller et al. 2004, Fitzpatrick et al. 1986 e Renehan et al. 1996) e outros não encontraram diferenças significantes entre ambos (Speight et al. 2002, Hocwald et al. 2001) 
Para tratamento de tumores malignos de glândulas salivares a cirurgia é o método de escolha, fazendo-se a ressecção completa com margem de segurança (Laurie e Licitra 2006, Day et al. 2004). A radioterapia adjuvante tem sido usada com o objetivo de reduzir a taxa de recorrência locorregional (Harrison et al. 1990, Terhaard et al. 2004). As indicações atuais da radioterapia adjuvante são: doença localmente avançada, margens cirúrgicas comprometidas, carcinomas de alto grau, infiltração perineural e metástase linfonodal. Terhaard et al. (2004) mostraram que a cirurgia associada à radioterapia pós-operatória aumenta a chance de controle locorregional quando comparada à cirurgia isoladamente, porém não altera a sobrevida. A principal recorrência após 10 anos são metástases a distância. Pohar et al. (2005) revisaram 163 carcinomas de parótida submetidos a tratamento cirúrgico com ou sem radioterapia. Este estudo também encontrou melhora do controle locorregional em pacientes tratados com cirurgia e radioterapia adjuvante. Houve recorrência locorregional ocorreu em $37 \%$ dos pacientes tratados somente por cirurgia e em $11 \%$ nos tratados por cirurgia e radioterapia adjuvante. A associação entre radioterapia e cirurgia reduziu o risco de recorrência locorregional, porém não alterou significativamente a sobrevida global. Garden et al. (1994), avaliando portadores de tumores de glândulas salivares menores descreveram recorrências locais em 19 pacientes (12\%), em seis em até 5 anos, em oito em 5 a 10 anos e em 5, após 10 anos. As complicações da radioterapia ocorreram em 51 pacientes (32\%). Terhaard et al. (2005) avaliaram 498 pacientes tratados com radioterapia isoladamente ou 
associada à cirurgia. A radioterapia associada à cirurgia melhorou o controle local em 10 anos, em portadores de tumores T3 e T4, casos em que a ressecção foi incompleta, havia invasão óssea ou invasão perineural e em casos com metástases cervicais.

North et al. (1990) analisaram 87 pacientes com carcinoma de glândulas salivares tratados com cirurgia com ou sem radioterapia adjuvante. A paralisia facial e a radioterapia prévia foram fatores de predição de recorrência local. Dezoito deles $(20,1 \%)$ apresentavam doença recorrente que foi tratada por cirurgia (4 casos; $22 \%$ ) e cirurgia com radioterapia (14; $78 \%)$. Houve falha no controle local em todos os pacientes tratados somente por cirurgia e $21 \%(3 / 14)$ nos tratados por cirurgia e radioterapia. Todas as recidivas foram no campo de radioterapia. A cirurgia com radioterapia adjuvante foi associada a melhor prognóstico para o controle locorregional em relação à cirurgia isoladamente.

Nos últimos anos alguns trabalhos mostraram boas taxas de controle locorregional, em tumores de glândulas salivares irressecáveis, utilizando irradiação com nêutrons rápidos e fótons. Saroja et al. (1998) realizaram a re-irradiação, com nêutrons rápidos, de pacientes com tumores recidivados, submetidos anteriormente à radioterapia radical. Foram tratados 10 pacientes portadores de tumores de glândulas salivares, oito pacientes responderam ao tratamento. Um paciente com carcinoma adenocístico, estava vivo sem doença, sete anos depois. Duncan et al. (1987) avaliaram 28 portadores de carcinomas de glândulas salivares recidivados ou com doença residual, tratados por radioterapia com nêutrons. Oito pacientes 
responderam ao tratamento inicial; a sobrevida variou de 1 a 84 meses com mediana de 19,5 meses. Douglas et al. (1996) utilizaram radioterapia por nêutrons para tratamento de pacientes portadores de carcinomas de glândulas salivares menores irressecáveis. Foram tratados 72 pacientes com intenção curativa; a sobrevida global em 5 anos foi de $59 \%$.

Wang et al. (1991) estudaram 24 pacientes com carcinoma de glândulas salivares irressecáveis tratados por radioterapia de fótons. Nos pacientes portadores de tumores de parótida a sobrevida global em 5 anos foi de $65 \%$ e o controle local foi de $100 \%$. Nos pacientes portadores de tumores de glândulas salivares menores, o controle local em 5 anos foi de $78 \%$ e a sobrevida de $93 \%$. Huber et al. (2001) avaliaram 75 portadores de carcinoma adenocísitico inoperável, tratados por radioterapia de neutrons, fótons e combinada. A taxa de controle local foi de $75 \%$ naqueles tratados com radioterapia de nêutrons e de $32 \%$ nos tratados com fótons ou combinada $(p=0,015)$. Esta diferença não influenciou a sobrevida. Os efeitos colaterais foram mais graves no tratamento por nêutrons (19\%), seguido pelo combinado (10\%) e pelos fótons (4\%). Griffin et al. (1988) realizaram um estudo prospectivo e aleatório em 32 portadores de tumores de glândulas salivares inoperáveis e com doença recidivada; 17 foram tratados com nêutrons (39\% dos pacientes com recorrência) e 15 com fótons (25\% daqueles com doença recorrente). A resposta foi completa em $85 \%$ dos pacientes tratados com nêutrons e em $33 \%$ dos tratados por fótons. A sobrevida em 2 anos foi $67 \%$ nos tratados com nêutrons e $17 \%$ nos tratados por fótons $(p=0,10)$. 
Metástases em linfonodos cervicais ocorrem em 13 a $16 \%$ dos casos (Spiro et al. 1989, Zbaren et al. 2003), sendo um importante fator de prognóstico, reduzindo a sobrevida em 5 anos de 74 para 10\% (Ferlito et al. 2002). O tratamento do pescoço é rotineiro quando há nódulos clinicamente positivos (Pohar et al. 2005, Kirkbride et al. 2001), sendo o esvaziamento cervical geralmente seguido de radioterapia.

Em carcinoma epidermóide de vias aerodigestivas, o esvaziamento cervical eletivo (realizado em pacientes sem metástases cervicais clinicamente detectáveis) pode ser indicado se a porcentagem de metástase oculta for superior a 20\% (da Cruz Perez et al. 2004); porém, em carcinoma de glândulas salivares, esta prática não está estabelecida (Zbaren et al. 2003).

Fiorella et al. (2005) realizaram um estudo multicêntrico com 28 instituições: a conduta em pacientes com pescoço N0, foi o esvaziamento cervical seletivo em $49 \%$ e somente radioterapia em $19 \%$. Entretanto, a conduta de observação clínica apenas, foi realizada em $32 \%$ dos pacientes.

Armstrong et al. (1992), analisando esvaziamento cervical em carcinoma de glândulas salivares maiores, mostraram que em 12\% (47/407) havia metástases ocultas, recomendando-se o tratamento do pescoço em tumores de alto grau (no qual havia $49 \%$ dos pacientes com metástases ocultas, $p<0,0001)$ e em tumores com mais de $4 \mathrm{~cm}(20 \%$ dos casos com linfonodos positivos, $p<0,0001)$. Foi estimado o risco de metástase cervical oculta, em carcinoma epidermóide (41\%), adenocarcinoma (18\%) e carcinoma mucoepidermóide (14\%). O esvaziamento sugerido seria o dos 
níveis I, II e III (esvaziamento supra-omo-hióideo), pois a maioria das metástases se encontrava nesses níveis. Rinaldo et al. (2003) defenderam o esvaziamento dos níveis Ib, Ila, Ilb, III e também Va. Krause (1981) sugeriu biópsia linfonodal nos níveis I e II durante a cirurgia para ressecção do tumor: se a congelação fosse positiva, o esvaziamento cervical deveria ser realizado. Medina (1998) resumiu as indicações de esvaziamento eletivo em carcinoma de glândulas salivares: tumores de alto grau, tumores T3 e T4, tumores com diâmetro maior que $3 \mathrm{~cm}$, paralisia facial, idade superior a 54 anos, extensão extra-glândular e invasão peri-linfática. Estas indicações são as mesmas utilizadas para a radioterapia adjuvante, com algumas indicações adicionais: envolvimento do lobo profundo, invasão perineural e doença microscópica residual próxima do nervo facial. O autor concluiu que a cirurgia para tumores de glândulas salivares com características preditivas de metástases ocultas deve incluir ressecção do tumor primário e radioterapia pós-operatória incluindo a área operada e o campo cervical eletivamente.

Apesar do emprego de tratamento cirúrgico radical e da radioterapia adjuvante, há elevada taxa de recorrências locoregionais, variando de 12 a 35\% dos casos (Spiro 1986, Pires et al. 2004, Leverstein et al 1998, Zbaren et al. 2003), enquanto metástases a distância ocorrem em 12 a 37\% (Spiro 1986, Zbaren et al. 2003). O tratamento de pacientes com recorrência locorregional e metástases a distancia é controverso, havendo poucas publicações sobre o assunto (Harrison et al. 1990, Terhaard et al. 2004). De 
modo geral, esses pacientes são considerados intratáveis devido um suposto mau prognóstico (Regis de Brito Santos et al. 2001)

Spiro (1986) revisou uma série de 2.807 pacientes com tumores de glândulas salivares, sendo 1.278 malignos (45\%). O tumor mais freqüente foi o carcinoma mucoepidermoide (34\%), seguido por carcinoma adenocístico (22\%) e adenocarcinoma (18\%). Recorrências locorregionais acometeram $39 \%$, principalmente locais. Houve uma taxa de recorrência mais elevada nos pacientes com tumores de glândulas submandibulares e salivares menores (60 e 65\%, respectivamente). Metástases a distância ocorreram em $17 \%$ dos pacientes com carcinoma de parótida; $37 \%$ nas submandibulares e $24 \%$ nas salivares menores. Os fatores associados a melhor prognóstico foram: sítio primário (parótida), tipo histológico (carcinoma de células acinares), grau histológico (baixo grau: adenocarcinomas, carcinoma mucoepidermóide e carcinoma epidermóide) e estádio precoce.

Lima et al. (2005) avaliaram fatores prognósticos clínicos em 126 portadores de tumores malignos da parótida, tratados por cirurgia e/ou radioterapia. Vinte e 5 pacientes tiveram recorrência (19,7\%), 17 apresentaram recorrência local $(13,5 \%)$, quatro locorregional $(3,1 \%)$ e quatro regional $(3,1 \%)$. $\mathrm{Na}$ análise multivariada, os fatores relacionados a pior evolução foram: grau histológico, estadiamento T e estadiamento clínico.

Walhberg et al. (2002), estudando a sobrevida 2.465 pacientes com carcinoma de glândulas parótida ou submandibular, observaram maior sobrevida em 10 anos naqueles com carcinomas de células acinares (88\%), seguido de carcinoma mucoepidermóide (80\%) e carcinoma adenocístico 
(74\%). O pior prognóstico foi observado em casos de carcinoma indiferenciado (44\% de sobrevida em 10 anos). Não houve diferença significativa em relação às topografias. Quanto à idade, em carcinoma mucoepidermóide e adenocarcinoma houve melhores taxas de sobrevida de pacientes com menos de 50 anos. Não há relato adequado das recorrências.

$\mathrm{Na}$ experiência do Hospital A.C. Camargo foram realizados diversos estudos com tipos histológicos e fatores prognósticos de tumores de glândulas salivares.

Lopes et al. (1998) analisaram 128 carcinomas de glândulas salivares menores da cavidade oral; encontraram variação de idade de 6 a 96 anos, com mediana de 49,1 anos. O tipo histológico mais freqüente foi o carcinoma mucoepidermóide $(59,4 \%)$. Observou-se recorrência local em 7,5\% dos casos; regional em 3,8\% e a distância em 4,7\%. Os fatores relacionados ao prognóstico foram estádio $\mathrm{N}$, comprometimento ósseo, histologia do tumor, sexo e tipo de cirurgia. A sobrevida global em 5 e 10 anos foi de $80,4 \%$ e $62,5 \%$, respectivamente.

Regis de Brito Santos et al. (2001) estudaram os fatores de predição para metástases cervicais em pacientes com carcinomas de parótida tratados por cirurgia: observaram que tipo histológico (carcinoma mucoepidermóide de alto grau, adenocarcinoma, carcinoma indiferenciado e carcinoma espinocelular), estádio T (T3 e T4) e desmoplasia severa, foram fatores associados a aumento do risco para ocorrência de metástases em linfonodos regionais. A taxa de metástases ocultas foi de 22,2\% em pacientes com tumor em estádio T3 e T4 e tipo histológico de alto risco. 
Houve recorrência local em 20 pacientes $(13,8 \%)$ e recorrência regional homolateral em nove $(6,2 \%)$, em um intervalo de 34 a 153 meses, mediana de 37,8 meses. A recorrência cervical contralateral surgiu em apenas três casos (2\%), 17 pacientes (11,7\%) apresentaram metástases a distância.

Ribeiro et al. (2002) analisando 38 crianças portadoras de tumores de glândulas salivares, encontraram 27 com tumores malignos (71\%), sendo o carcinoma mucoepidermóide o mais freqüente (17 pacientes; 44,7\%).

sexo feminino foi o preponderante $(1,9: 1)$; a mediana da idade foi de 11,8 anos. A sobrevida global em 5 anos foi de $81,6 \%$. O grau de diferenciação foi o único fator prognóstico nos pacientes com carcinoma mucoepidermóide; a sobrevida em 5 anos para casos de baixo grau e intermediário ou alto grau foi 100 e $50 \%$, respectivamente $(p=0,01)$. $O$ tempo de seguimento variou de dois meses a 40,3 anos, com mediana de 12,7 anos. Sete pacientes apresentaram recorrência (18,4\%): sendo locorregional em quatro casos, local em um e a distância em dois. Da Cruz Peres et al. (2004) estudaram 53 pacientes com idade inferior a 18 anos, portadores de tumores de glândulas salivares. Vinte e seis (49\%) apresentavam tumores malignos, sendo o mais comum o carcinoma mucoepidermóide. Recorrência local e/ ou regional foi observada em 11,5\% dos casos, com seguimento médio de 14,3 anos. Analisaram-se a expressão de p53, Ki-67, c-erbB-2, bcl-2 e CEA porém não foram marcadores preditivos.

Etges et al. (2003) estudando o carcinoma de ducto salivar, encontrou uma relação entre a hiperexpressão do c-erbB2 e a agressividade do tumor. 
Pires et al. (2004) mostraram que em pacientes com carcinoma mucoepidermóide de glândulas salivares, os fatores associados a um pior prognóstico em análise multivariada são: idade superior a 40 anos, estádios clínicos III e IV, presença de linfonodos positivos, fixação do tumor a estruturas adjacentes e alto grau histológico. Houve recorrência local em $12 \%$ dos pacientes estudados, com intervalo de 2 a 207 meses (mediana de 41,7 meses).

Alves et al. (2004) estudaram a expressão de Ki-67, PCNA (marcadores de proliferação celular) e p53 em tumores malignos (mucoepidermóide e carcinoma adenóide cístico) e benignos (adenoma pleomórfico) de glândula submandibular. Todos os tumores malignos foram positivos para PCNA e todos os adenomas pleomórficos foram negativos para Ki-67 e p53.

Da Cruz Peres et al. (2006) avaliaram fatores prognósticos clínicos, anatomopatológicos e de marcadores de carcinoma adenocístico em 129 pacientes. A idade variou de 10 a 96 anos, com mediana de 51,5. Os pacientes foram tratados por cirurgia isoladamente $(30,2 \%)$ e cirurgia com radioterapia adjuvante $(42,7 \%)$. O total de casos com recorrência (local e /ou regional e a distância) foi 71 (55\%) e o tempo para ocorrência de recidiva variou de 1 a 109 meses com mediana de 27,2. O tipo de recorrência foi a distância em 74,7\% (associada ou não a recorrência local e lou regional), local em $19,7 \%$, regional em $2,8 \%$ e locorregional em 2,8\%. Na análise multivariada, estádio clínico $(p=0,004)$, tipo histológico (sólido) $(p<0,001)$ e hiperexpressão de p53 $(p=0,05)$ foram fatores prognósticos independentes. 
Apesar da elevada incidência de recorrências loco-regionais, na literatura há poucos trabalhos que abordam a cirurgia de resgate em carcinomas de glândulas salivares. Todos citam dados dos pacientes recidivados que foram tratados por cirurgia de resgate, geralmente com resultados decepcionantes. Leverstein et al. (1998) analisaram 65 carcinomas de parótida. A recorrência locorregional apareceu em 12 casos (18\%), sendo submetidos a cirurgia de resgate. Somente um paciente sobreviveu 51 meses, morrendo de causas não relacionadas à doença. Os demais morreram com a doença em atividade. Oito pacientes apresentaram metástases a distância

Parsons et al. (1996) estudaram carcinoma de glândulas salivares menores em 87 pacientes sem tratamento prévio tratados por cirurgia com radioterapia adjuvante $(44,50,5 \%)$ e radioterapia isoladamente. Metástases a distância ocorreram em 31\% dos pacientes. Houve 25 recidivas locais. Apenas 5 pacientes foram submetidos à cirurgia (20\%), dos remanescentes 13 pacientes tinham tumores considerados irressecáveis, quatro apresentavam metástases a distância; dois recusaram a cirurgia e um tinha contra-indicações clínicas. Dos 5 pacientes operados, um morreu no pósoperatório, três tiveram recorrência precoce e somente um permaneceu livre de doença.

Tran et al. (1986) analisaram 133 portadores de carcinoma de glândulas salivares tratados por cirurgia com ou sem radioterapia. O tipo histológico mais freqüente foi o carcinoma mucoepidermóide (37\%), seguido pelo carcinoma adenocístico (22\%). Sessenta e oito pacientes foram 
tratados somente com cirurgia e 57 com cirurgia e radioterapia. Quarenta e seis pacientes tiveram recorrência, sendo 13 locais. A sobrevida dos pacientes que apresentaram recorrência local foi $23 \%$ em 5 anos.

Spiro et al. (1982) estudaram 204 pacientes com adenocarcinoma de glândulas salivares e identificaram 187 com informação adequada, quanto à recorrência. Noventa e 5 pacientes apresentaram recorrência local (51\%). O risco de recorrência foi maior que $70 \%$ nos portadores de tumores da cavidade nasal, seios paranasais e tumores grau III. Apesar do tratamento aplicado a essas recidivas, somente 12 pacientes $(12,6 \%)$ tiveram resposta.

Terhaard et al. (2004) avaliaram 595 portadores de carcinoma de glândulas salivares e observaram que 100 (16,8\%) tiveram recorrência ou doença residual. Destes, somente 46 foram submetidos a tratamento de resgate (não há descrição do tratamento utilizado), obtendo-se controle da doença em somente 17 pacientes ( $37 \%$ dos submetidos ao tratamento de resgate). O seguimento foi de 4 a 128 meses com mediana de 58 . Houve recorrência ou doença residual regional em 61 pacientes $(10,2 \%)$, sendo que 39 foram submetidos ao tratamento de resgate. Houve controle da doença em 15 pacientes $(38,5 \%$ dos submetidos ao tratamento de resgate). $O$ seguimento variou de 11 a 123 meses com mediana de 67.

O papel da quimioterapia no tratamento não está definido, sendo seu uso geralmente em doença metastática ou irressecável ou em protocolos de investigação (Laurie e Licitra 2006, Harish 2004). O trabalhos existentes compreendem séries com pequeno número de pacientes, que mostraram 
uma taxa de resposta objetiva de 15 a $44 \%$ e duração média de resposta de apenas 6 meses (Laurie e Licitra 2006, Airoldi et al. 1989 e 2000).

Em uma revisão sobre o assunto são citados vários estudos utilizando os mais diferentes esquemas de quimioterapia (Licitra et al. 1996): cisplatina isoladamente (associada a adriamicina, epirrubicina, 5-Fluorouracil, vinovelbine), paclitaxel isoladamente ou associada, mitoxantrone e outros.

Suen e Johns (1982) analisaram 85 pacientes portadores de carcinoma de glândulas salivares tratados em 10 instituições por quimioterapia. Os tipos histológicos mais freqüentes foram carcinoma adenocístico $(63,2 \%)$ e adenocarcinoma $(11,8 \%)$, geralmente tratados por cisplatina ou 5-Fluorouracil,, isoladamente ou em associações. A taxa de resposta (completa e parcial) foi 39,6 e 50\%, respectivamente.

Airoldi et al. (2000) realizaram ensaio clínico em tumores de glândulas salivares maiores e menores, empregando paclitaxel e carboplatina. Verificaram resposta parcial em $14 \%$ dos pacientes; porém em $50 \%$ não houve resposta e em $36 \%$ houve progressão da doença. E outro estudo Airoldi et al. (2001) estudaram pacientes com doença recorrente e/ou metastática, tratados com cisplatina isoladamente ou cisplatina associada ao vinorelbine (um inibidor do mecanismo de polimerização de tubulina, que bloqueia a divisão celular nas fases $\mathrm{G} 2 / \mathrm{M})$. As melhores respostas ocorreram nos pacientes tratados com cisplatina associada ao vinorelbine: com resposta completa em $19 \%$ e parcial em $25 \%$ dos pacientes. Não houve diferença significante quanto à sobrevida nos dois grupos. 
Dreyfuss et al. (1987) estudaram 13 pacientes com doença recorrente e/ou metastática de glândulas salivares maiores e menores, tratados com cisplatina associada a ciclofosfamida e doxorrubicina. A resposta foi completa em três pacientes e parcial em três (taxa de $46 \%$ de resposta). A duração da resposta foi de apenas 5 meses (de 2 a 8 meses). Licitra et al. (1996) analisaram 22 portadores de carcinoma avançado de glândulas salivares, tratados com um esquema de cisplatina associada a ciclofosfamida e doxorrubicina, A dosagem foi igual à utilizada por Dreyfuss et al. (1987), porém com tempo mais longo, entre os ciclos (28 dias). Nenhum paciente teve resposta completa e $27 \%$ (seis pacientes) exibiram resposta parcial.

A quimioterapia aparentemente apresenta um resultado melhor em pacientes com doença local e/ou regional (Licitra et al. 1996, Suen e Johns 1982).

Gilbert et al. (2006), em um estudo fase II com paclitaxel em 45 portadores de carcinoma de glândulas salivares avançado, encontraram resposta parcial em 23,1\% dos carcinomas mucoepidermóides (três respostas parciais em 13 pacientes tratados) e $29,4 \%$ dos adenocarcinomas (5 respostas parciais em 17 pacientes). Taxa de resposta objetiva foi $26 \%$. Não houve resposta nos carcinomas adenocísticos. Nenhum paciente mostrou resposta completa e a sobrevida em 3 anos foi menos de $25 \%$. Entre os pacientes portadores de carcinoma mucoepidemóide, adenocarcinoma e carcinoma adenocístico, não houve diferença significante na sobrevida global. 
A tendência atual é realizar tratamento com base em alvos moleculares. Os tumores de glândulas salivares têm diversos marcadores em investigação: c-kit, receptor do fator de crescimento epidérmico (EGFR), p53 e outros (Airoldi et al. 2003). Vered et al. (2002) analisaram EGFR em 27 amostras de carcinoma adenóide cístico, encontrando 23 amostras (85\%), positivas para EGFR.

Gallo et al. (1995) estudaram a expressão do gene TP53 mutado em carcinoma de glândulas salivares e encontraram hiperexpressão em $67 \%$ das amostras. Esta alteração associou-se ao prognóstico: quanto mais intensa a expressão, pior o prognóstico em relação à sobrevida global.

A proteína c-kit é um receptor tipo tirosina quinase, importante no desenvolvimento de células hematopoiéticas e melanócitos. A hiperexpressão de c-kit foi identificada em 80 a $90 \%$ das amostras de carcinoma adenocístico testadas (Jeng et al. 2000 e Holst et al. 1999). O mesilato de imatinib é um potente inibidor da proteína quinase c-kit e foi utilizado em estudos clínicos para tratamento de carcinoma adenocístico avançado. Alcedo et al. (2004) relataram dois casos de pacientes portadores de carcinoma adenocístico de glândula submandibular e base da língua, tratados com mesilato de Imatinib. O paciente portador de carcinoma de tumor de glândula submandibular mostrou resposta completa, não havendo evidência de doença 20 meses após o tratamento, o outro teve resposta parcial, permanecendo com doença estável 15 meses após o tratamento. Apesar desse resultado animador, Hotte et al. (2005) realizando um estudo fase II, com 15 portadores de carcinoma adenóide cístico, com 
hiperexpressão de c-kit, tratados com mesilato de imatinib, não obtiveram os mesmos resultados. Nenhum paciente exibiu resposta objetiva, em nove deles a doença permaneceu estável e, em seis, progrediu. O estudo foi cancelado após a primeira fase. Novos estudos são necessários para estabelecer o real papel do mesilato de imatinib no tratamento da carcinoma adenocístico.

Há estudos prospectivos em andamento, testando diversos esquemas de quimioterapia (gencitabina isoladamente ou combinada com cisplatina, capecitabina mais oxiplatina e outros), porém a grande variabilidade histológica e a relativa raridade da doenca tornam esses estudos de difícil execução (Laurie e Licitra 2006).

Os pacientes que apresentam recidivas locoregionais de carcinoma de glândulas salivares são desafiadores para o cirurgião de cabeça e pescoço e o oncologista. A decisão terapêutica de resgate diante desses pacientes é difícil. Há uma tênue linha entre o benefício em termos de sobrevida que pode ser oferecida pelos tratamentos utilizados atualmente (cirurgia e/ou radioterapia) de um lado e as seqüelas, de outro. As decisões freqüentemente são baseadas em experiências pessoais e não em dados objetivos da literatura.

Portanto, avaliar resultados tardios e definir fatores prognósticos com base na experiência de um centro de referência poderá ser útil ao estabelecimento de critérios para a indicação de tratamento de resgate curativo ou paliativo. 
OBJETIVOS 


\section{OBJETIVOS}

Esse estudo tem como objetivo principal identificar fatores prognósticos clínicos, anatomopatológicos e terapêuticos em pacientes portadores de carcinomas recidivados de glândulas salivares submetidos a cirurgia de resgate.

O objetivo secundário é descrever complicações e mortalidade operatória nos pacientes estudados. 
CASUÍSTICA E MÉTODOS 


\section{CASUÍSTICA E MÉTODOS}

Foram revisados os prontuários de todos os pacientes portadores de carcinomas de glândulas salivares recidivados, tratados Hospital do Câncer A.C. Camargo, no período de 1957 a 2000. A fonte das informações coletadas foram o arquivo do Departamento de Anatomia Patológica (contendo todos os laudos de exames anatomo patológicos das peças cirúrgicas de pacientes com tumores de glândulas salivares) e o registro de procedimentos cirúrgicos realizados no período.

\subsection{Critérios de Elegibilidade}

Foram considerados elegíveis para o estudo os pacientes que preenchiam os seguintes critérios:

1- Submetidos a tratamento prévio com cirurgia ou radioterapia ou combinação dessas modalidades terapêuticas, com recorrências locais e/ou regionais;

2- Sem evidência de metástases a distância;

3- Submetidos a cirurgia de resgate com intenção curativa;

4- Dados registrados nos prontuários que possibilitassem 0 estadiamento clínico do tumor recorrente.

Identificaram-se 59 pacientes que preenchiam os critérios de elegibilidade estabelecidos para o estudo. Com base em dados do prontuário médico, foi preenchida a ficha com dados demográficos, clínicos, anatomopatológicos e terapêuticos (anexo 1). Dentre estes casos, 35 pacientes $(59,3 \%)$ haviam sido tratados previamente em outra instituição. 


\subsection{Dados demográficos}

Trinta e quatro pacientes $(57,6 \%)$ eram do sexo masculino e 25 $(42,4 \%)$ do sexo feminino; $50(84,7 \%)$ eram da raça branca e $9(15,3 \%)$ não branca. A idade, no momento da cirurgia de resgate, variou de 10 a 75 anos, com mediana de 51 anos.

\subsection{Dados relativos ao tumor primário e ao tipo histológico}

O local do tumor primário foi a parótida, em 34 casos $(57,6 \%)$, cavidade oral em 13 casos $(22,1 \%)$ e glândula submandibular em 12 casos $(20,3 \%)$ (Figura 1).

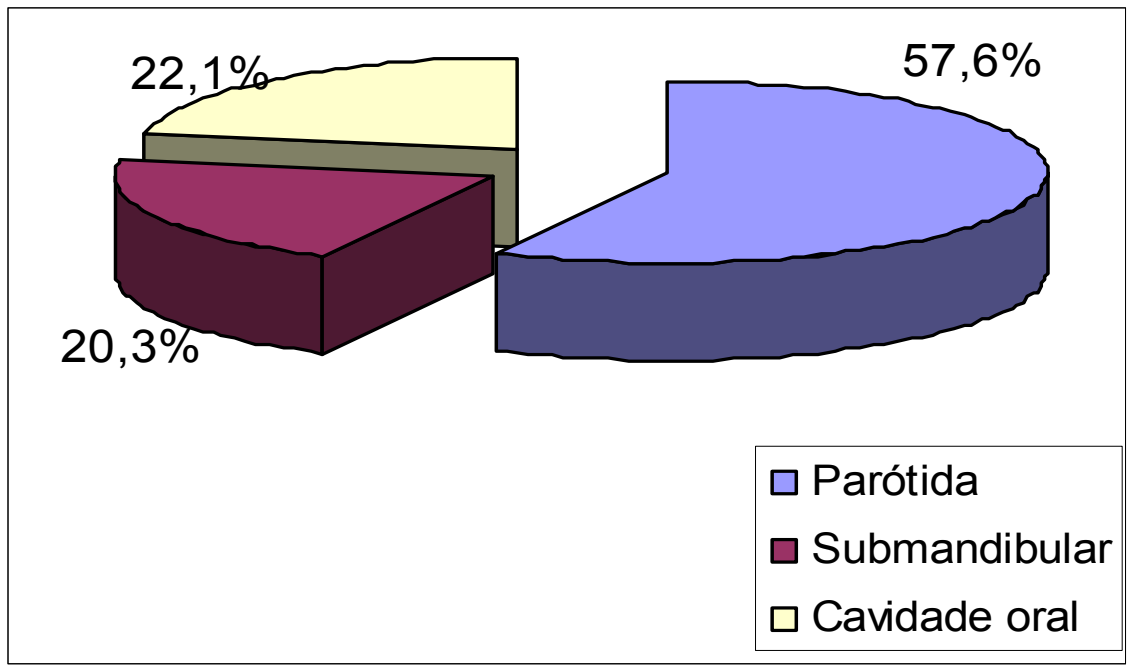

Figura 1 - Distribuição dos casos conforme a localização do tumor primário.

A classificação histológica utilizada foi a da Organização Mundial de Saúde de 1992 (Seifert 1992), na qual há divisão em 16 tipos histológicose outros: carcinoma adenocístico, carcinoma mucoepidermóide, carcinoma de células acinares, carcinoma epidermóide, adenocarcinoma polimórfico de 
baixo grau, adenocarcinoma de células basais, carcinoma sebáceo, cistadenocarcinoma papilífero, adenocarcinoma mucinoso, carcinoma oncocítico, carcinoma de ductos salivares, adenocarcinoma, carcinoma epitelial-mioepitelial (mioepitelioma maligno), carcinoma ex-adenoma pleomórfico, carcinoma de pequenas células, carcinoma indiferenciado e outros. O tipo histológico mais freqüente foi o carcinoma adenocístico em 23 casos (39\%), seguido pelo carcinoma mucoepidermóide com 14 (23,7\%) (Tabela 1). 
Tabela 1. Distribuição dos casos de acordo com o tipo histológico e o local do tumor primário.

\begin{tabular}{lrrrr}
\hline & \multicolumn{3}{c}{ Topografia } \\
Tipo histológico & $\begin{array}{c}\text { Parótida } \\
\mathrm{n}(\%)\end{array}$ & $\begin{array}{c}\text { Submandibular } \\
\mathrm{n}(\%)\end{array}$ & $\begin{array}{c}\text { Cavidade oral } \\
\mathrm{n}(\%)\end{array}$ & Total \\
\hline Carcinoma mucoepidermóide & $13(38,2)$ & $1(8,3)$ & 0 & $\mathbf{1 4}$ \\
Carcinoma adenocístico & $9(26,5)$ & $6(50,0)$ & $8(61,5)$ & $\mathbf{2 3}$ \\
Adenocarcinoma & $2(5,9)$ & $1(8,3)$ & $3(23,1)$ & $\mathbf{6}$ \\
Carcinoma epidermóide & $1(2,9)$ & $2(16,8)$ & 0 & $\mathbf{3}$ \\
Carcinoma indiferenciado & 0 & $1(8,3)$ & 0 & $\mathbf{1}$ \\
Carcinoma de cel acinares & $4(11,7)$ & 0 & $1(7,7)$ & $\mathbf{5}$ \\
Carcinoma ex-adenoma & $3(8,8)$ & 0 & 0 & $\mathbf{3}$ \\
pleomórfico & $2(5,9)$ & $1(8,3)$ & $1(7,7)$ & $\mathbf{4}$ \\
Outros & $\mathbf{3 4}(\mathbf{1 0 0 , 0 0 )}$ & $\mathbf{1 2}(\mathbf{1 0 0 , 0 0})$ & $\mathbf{1 3}(\mathbf{1 0 0 , 0 0})$ & $\mathbf{5 9}$ \\
Total & & & & 0 \\
\hline
\end{tabular}

\subsection{Dados relativos ao tratamento prévio e às recorrências}

O tratamento prévio foi resseção do tumor primário em 52 pacientes (88\%) e ressecção com esvaziamento cervical em sete pacientes (12\%). A radioterapia adjuvante foi aplicada em 11 pacientes (18,7\%) e quimioterapia adjuvante, em um paciente.

As recorrências foram agrupadas como locais, regionais (linfonodos cervicais) e locorregionais. A maioria dos pacientes apresentou recorrência da doença no local do tumor primário (39; 66\%), em sete casos a recorrência foi regional $(11,9 \%)$ e, em treze, casos foi locorregional $(22,1 \%)$ (Figura 2). 


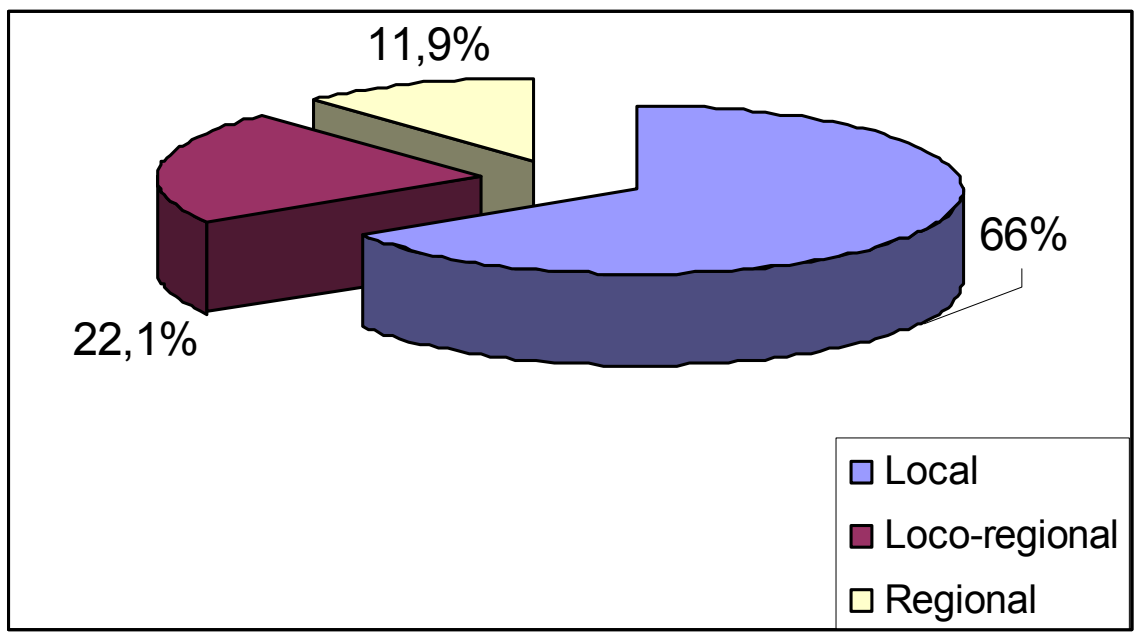

Figura 2. Distribuição dos casos de acordo com tipo da recorrência.

Dentre os pacientes submetidos à cirurgia e a esvaziamento cervical, dois apresentaram recidiva regional, porém no pescoço contralateral. Não houve recidiva cervical na área do esvaziamento. Nos submetidos a radioterapia, não houve recidiva locorregional. A Figura 3 descreve o tipo de recidiva conforme o tratamento prévio (com ou sem radioterapia adjuvante).

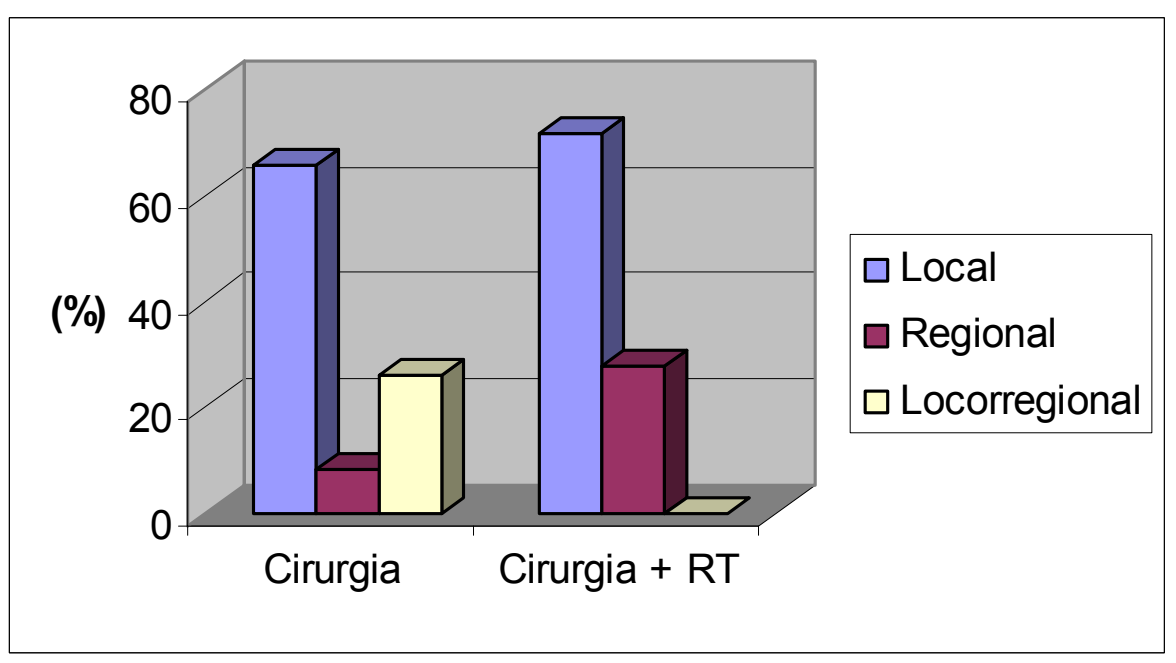

Figura 3. Sítio de recorrência conforme tratamento prévio. 
As recorrências em portadores de tumores primários da parótida foram, em sua maioria locais $(67,6 \%)$, seguidas pelas recorrências locorregionais $(26,6)$ e regionais $(5,8 \%)$ (Figura 4$)$.

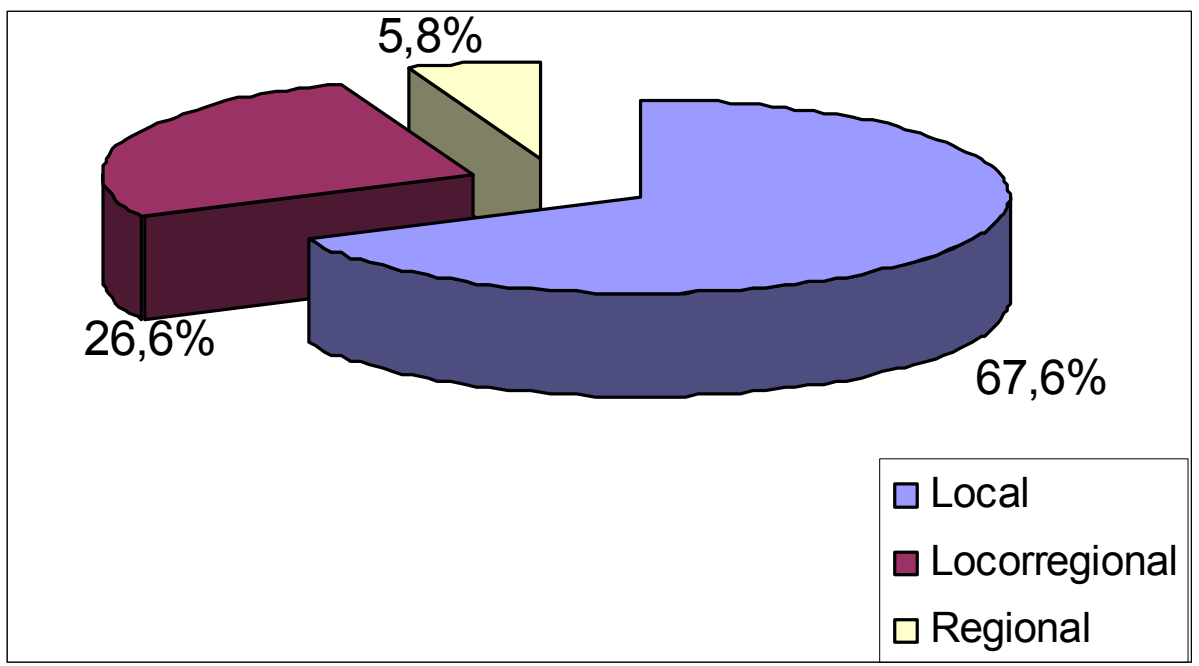

Figura 4. Distribuição dos sítios de recorrência dos pacientes com tumores da parótida.

Nos pacientes com tumores da glândula submandibular, as recorrências mais freqüentes foram locais $(58,3 \%)$, seguidas pelas locorregionais (25\%) e regionais (16,7\%) (Figura 5). 


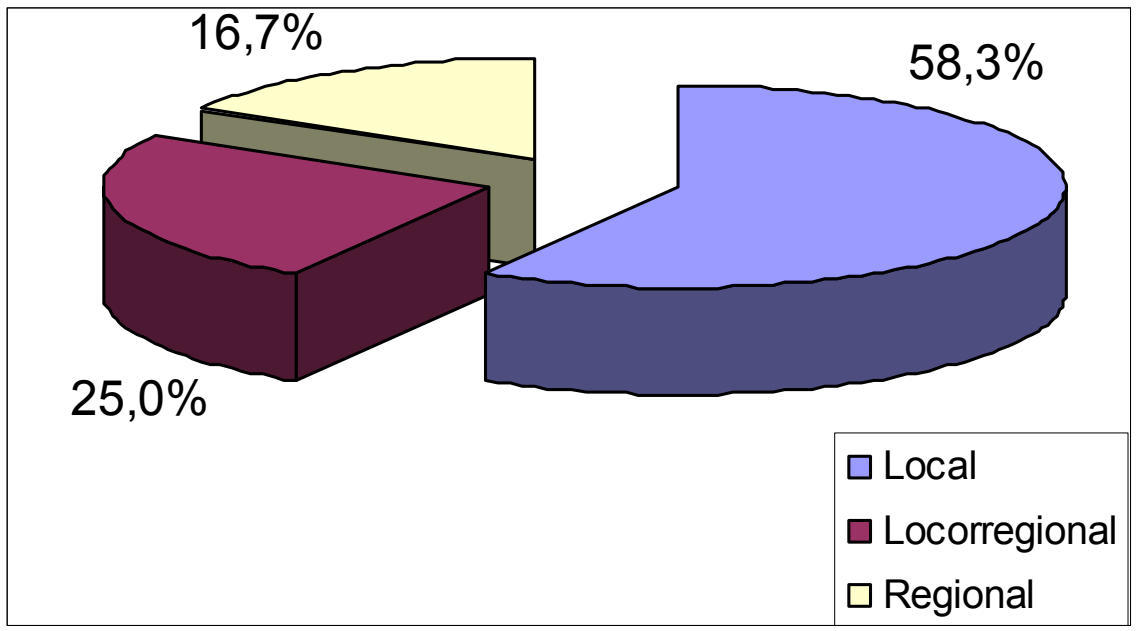

Figura 5. Distribuição dos sítios das recorrências em pacientes com tumores da glândula submandibular.

Nos tumores originários das glândulas salivares menores localizados na cavidade oral, as recorrências mais freqüentes foram as locais (69,5\%), seguidas pelas regionais $(23,7 \%)$ (Figura 6).

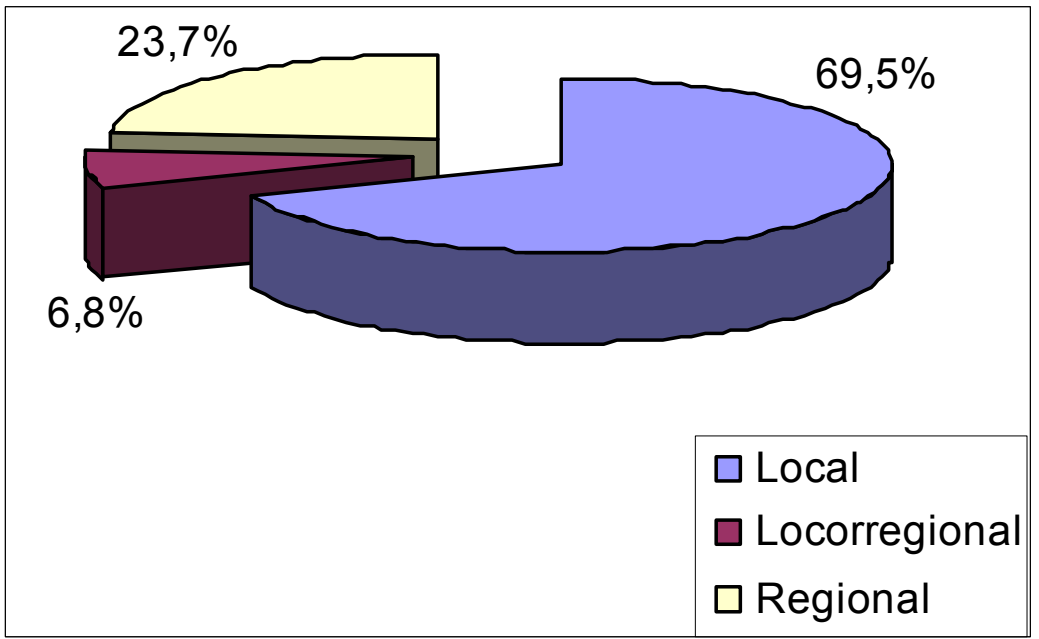

Figura 6. Distribuição dos sítios de recorrência de tumores de glândulas salivares menores intra-orais.

Todos os pacientes tiveram a recorrência tumoral estadiada (rTNM) de acordo com a classificação da AJCC de 2002, com base nos dados clínicos pré-operatórios disponíveis nos prontuários médicos, na descrição do exame 
locorregional e no planejamento terapêutico. Sempre que disponíveis, também foram utilizados dados obtidos de exames de diagnóstico por imagem, como raios $\mathrm{X}$, tomografia computadorizada e ressonância nuclear magnética. O estádio clínico da recorrência foi inicial (rEC I e II) em 15 casos $(25,5 \%)$ e avançado (rEC III e IV) em 44 (74,5\%). Houve seis casos de rEC I e nove de rEC II. Trinta e 5 pacientes (59,3\%) tinham tumores estadiados como rEC IV (Figura 7).

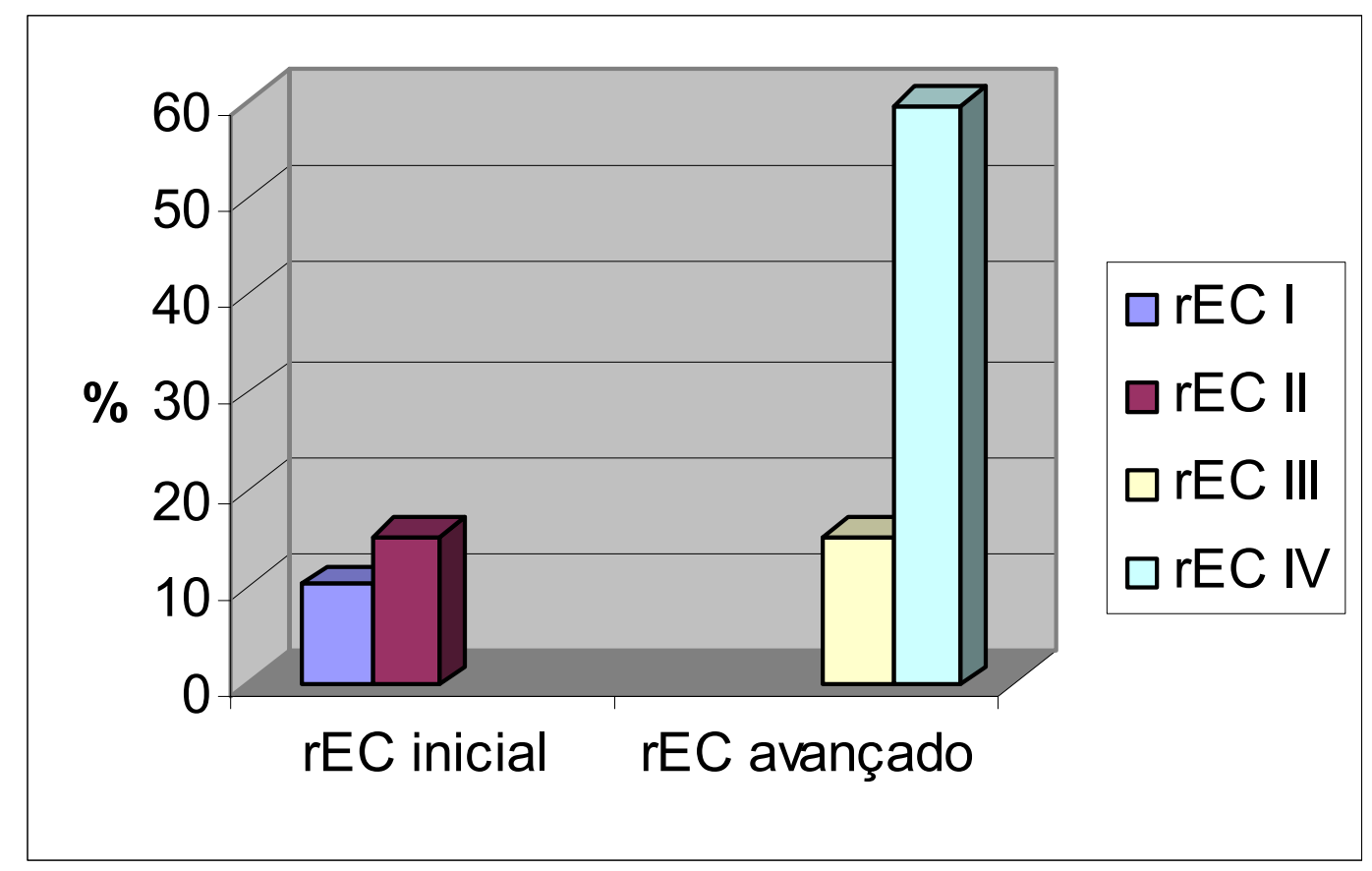

Figura 7. Distribuição dos casos segundo o estádio clínico da recorrência tumoral (rTNM)

O tempo entre a primeira cirurgia e a cirurgia de resgate variou de 2,6 a 78,4 meses com mediana de 20,9. Mais da metade da recorrência dos tumores foi diagnosticada antes um anos após o tratamento inicial. (Figura 8) 


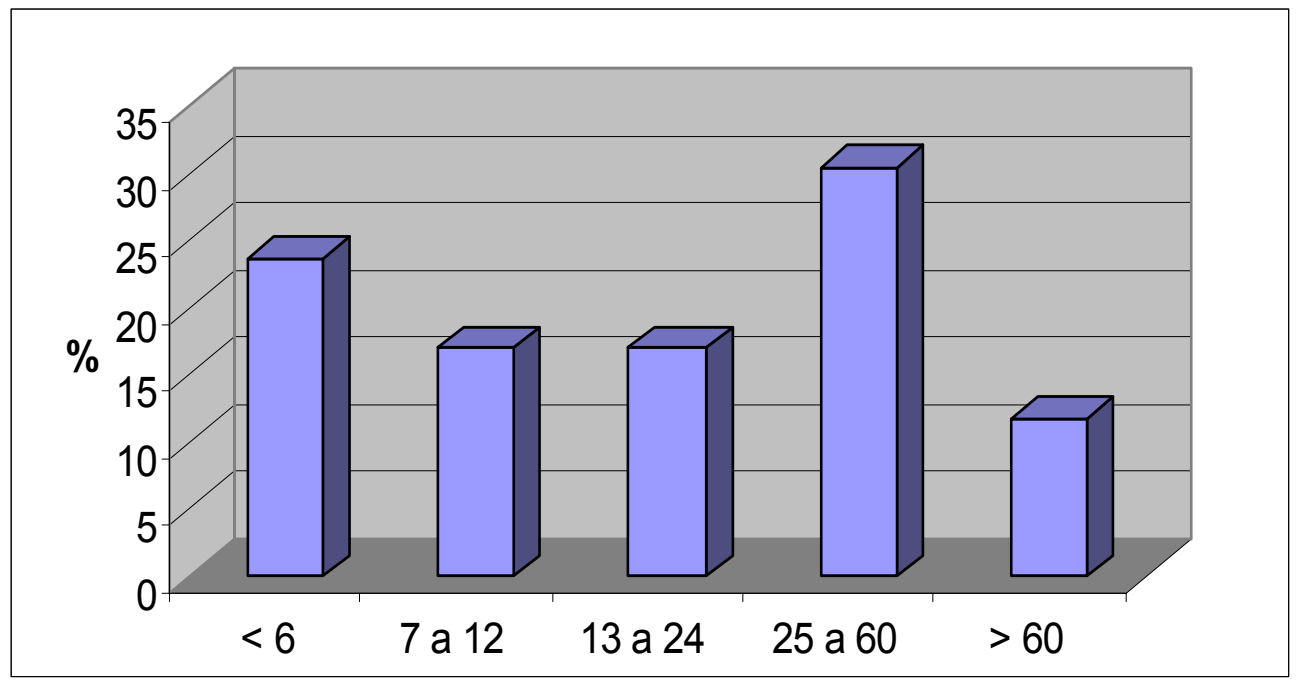

Figura 8. Distribuição do tempo entre o tratamento inicial e a recorrência, em períodos (meses).

O tratamento cirúrgico da recorrência foi ressecção local em 39 casos $(66,1 \%)$, esvaziamento cervical isolado em 7 casos $(11,9 \%)$ e cirurgias em monobloco (ressecção local associada a esvaziamento cervical) em 13 casos (22\%). Os tipos de cirurgia são mostrados na tabela 2. 
Tabela 2. Tratamento cirúrgico da recorrência local, regional ou locorregional.

\begin{tabular}{|c|c|c|}
\hline \multirow[t]{2}{*}{ Tipo de cirurgia } & \multicolumn{2}{|c|}{ Esvaziamento cervical } \\
\hline & Não & Sim \\
\hline $\begin{array}{l}\text { Parotidectomia total com conservação do } \\
\text { nervo facial }\end{array}$ & 4 & 1 \\
\hline Parotidectomia total & 2 & 0 \\
\hline Parotidectomia total ampliada & 10 & 7 \\
\hline $\begin{array}{l}\text { Parotidectomia total ampliada com } \\
\text { ressecção do osso temporal }\end{array}$ & 5 & 0 \\
\hline $\begin{array}{l}\text { Parotidectomia total ampliada com } \\
\text { mandibulectomia }\end{array}$ & 1 & 1 \\
\hline $\begin{array}{l}\text { Ressecção ampliada da região } \\
\text { submandibular }\end{array}$ & 7 & 2 \\
\hline $\begin{array}{l}\text { Ressecção de lesão oral sem } \\
\text { mandibulectomia }\end{array}$ & 3 & 0 \\
\hline $\begin{array}{l}\text { Ressecção de lesão oral com } \\
\text { mandibulectomia }\end{array}$ & 1 & 2 \\
\hline Maxilectomia & 3 & 0 \\
\hline Ressecção craniofacial & 2 & 0 \\
\hline Ressecção em orofaringe & 1 & 0 \\
\hline $\begin{array}{l}\text { Esvaziamento cervical } \\
\text { (recorrência regional) }\end{array}$ & & 7 \\
\hline
\end{tabular}

Todas as cirurgias de resgate foram ressecções radicais, na opinião do cirurgião ou pela comprovação com exames anatomopatológicos das peças cirúrgicas. 
A reparação dos defeitos cirúrgicos foi feita com fechamento primário em 30 casos $(50,8 \%)$, retalho local em $15(25,4 \%)$, enxerto livre de pele em $3(5,2 \%)$. Em onze casos $(18,6 \%)$ foram utilizados retalhos miocutâneos ou transplantes microcirúrgicos devido à complexidade da reparação do defeito cirúrgico. (Figura 9).

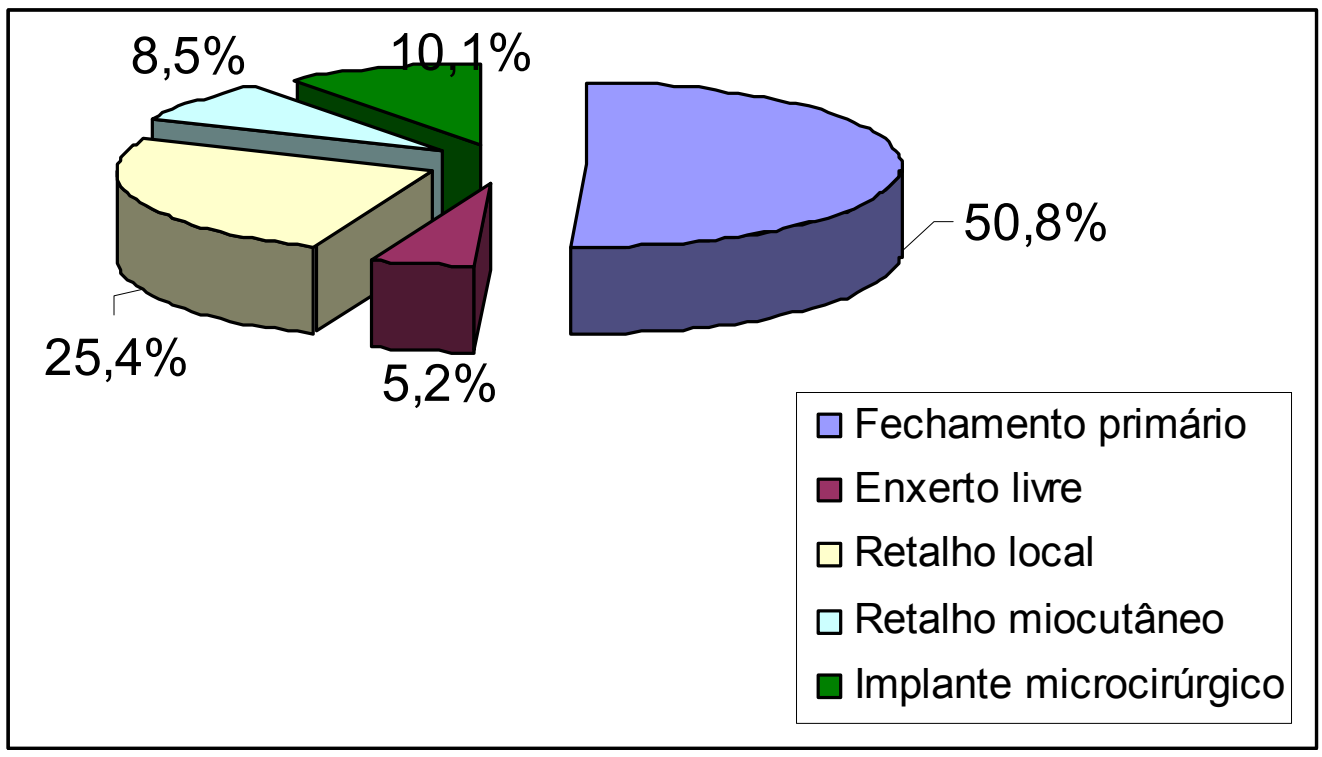

Figura 9. Tipos de reconstrução na cirurgia de resgate.

A radioterapia adjuvante foi possível em 34 casos $(57,6 \%)$. 5 pacientes foram submetidos à re-irradiação, a maioria deles havia recebido radioterapia somente para o tumor primário. Nesses casos foram usadas doses menores que as habitualmente utilizadas para tratamento radical. 


\subsection{Complicações e Óbitos}

As complicações foram divididas em locais e sistêmicas. As locais incluíram: infecção da ferida operatória, paralisia facial (quando o nervo havia sido preservado), seroma, hematoma, necrose de retalhos e outros. As sistêmicas foram: broncopneumia, infarto agudo do miocárdio, acidente vascular cerebral e outros.

A morte pós-operatória foi definida como a morte ocorrida até trinta dias após a cirurgia de resgate.

\subsection{Análise Estatística}

Para a análise estatística foi utilizado o programa estatístico STATA versão 7.0. A análise de sobrevida atuarial foi realizada pelo método de Kaplan-Meier. O tempo de sobrevida global foi definido como o intervalo entre a data do tratamento de resgate e a última informação objetiva de seguimento ou a morte do paciente. Para cálculo da significância estatística entre os resultados obtidos para as diferentes categorias de uma mesma variável empregou-se o teste log-Rank. Significância estatística foi determinada por valores de $p<0,05$ 


\section{RESULTADOS}

\subsection{Complicações}

Dezoito pacientes sofreram complicações locais após a cirurgia de resgate $(30,5 \%)$ e um deles apresentou complicação sistêmica (confusão mental temporária)(1,7\%). Taxa de complicação global foi de $32,2 \%$. As complicações locais mais freqüentes foram infecção da ferida operatória $(8,5 \%)$ e paralisia facial $(6,7 \%)$. Três pacientes exibiram mais de uma complicação: dois com infecção da ferida operatória e necrose de retalhos e um com infecção da ferida operatória e fístula orocutânea. Não houve morte pós-operatória. Na tabela 3 descrevem-se as complicações locais.

Tabela 3. Tipo de complicações locais após cirurgia de resgate para tumor de glândulas salivares.

\begin{tabular}{ll}
\hline \multicolumn{1}{c}{ Tipo de complicação } & $\mathrm{n}(\%)$ \\
\hline Infecção da ferida operatória & $5(8,5)$ \\
Paralisia facial & $4(6,7)$ \\
Seroma & $2(3,4)$ \\
Hematoma & $1(1,7)$ \\
Necrose de retalhos & $2(3,4)$ \\
Síndrome de Frey & $1(1,7)$ \\
Fístula orocutânea & $1(1,7)$ \\
Infecção e necrose de retalho & $2(3,4)$ \\
Infecção e fístula & $1(1,7)$ \\
\hline
\end{tabular}




\subsection{Sobrevida Atuarial Global}

A sobrevida global após a cirurgia de resgate foi $61,3 \%$ em 5 anos e $42 \%$ em 10 anos (Figura 10). Vinte e três pacientes (39\%) não apresentaram qualquer evidência de recorrência até a última informação no prontuário. $O$ seguimento variou de 24 dias a 34,6 anos, com mediana de 6,7 anos. Oito pacientes $(13,6 \%)$ foram perdidos de seguimento.

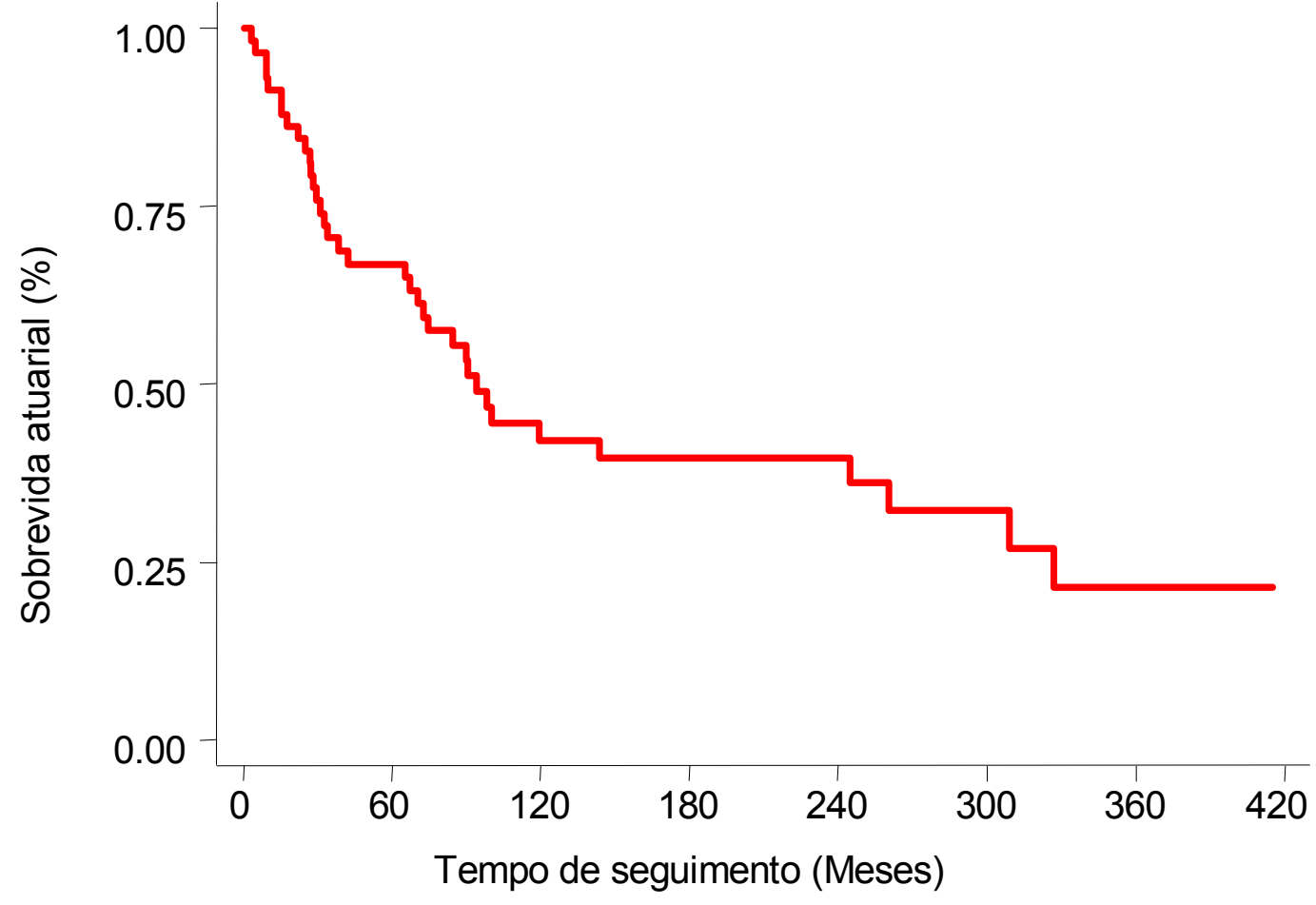

Figura 10. Curva de sobrevida atuarial global dos 59 pacientes submetidos à cirurgia de resgate por carcinomas de glândulas salivares recidivados. 


\subsection{Recorrência após a cirurgia de resgate}

Houve nova recorrência em 36 pacientes (61\%). A recorrência mais freqüente foi a distância, que ocorreu em 16 casos (27\%). O pulmão foi o local mais comum dessas metástases. Em 10 casos $(17 \%)$ houve falha no controle local e em 5 casos (8,5\%), falha locorregional. As falhas no pescoço, tanto ipsilaterais quanto contralaterais, aconteceram em 5 casos (8,5\%). O tempo para ocorrência da recidiva após a cirurgia de resgate variou de 2,8 meses a 26,6 anos, com mediana de 2,3 anos. A tabela 4 relaciona o tipo de recorrência submetida à cirurgia de resgate com o local da nova recorrência. 
Tabela 4. Distribuição da segunda recorrência de acordo com o sitio da primeira recorrência.

\begin{tabular}{|c|c|c|c|c|}
\hline Primeira recorrência & $\mathrm{n}$ & Segunda recorrência & $\mathrm{n}$ & $(\%)$ \\
\hline \multirow[t]{5}{*}{ Local } & 39 & Não & 14 & $(35,9)$ \\
\hline & & Local & 6 & $(25,4)$ \\
\hline & & Regional & 3 & $(7,7)$ \\
\hline & & Locorregional & 4 & $(10,2)$ \\
\hline & & Distância & 12 & $(30,8)$ \\
\hline \multirow[t]{4}{*}{ Regional } & 7 & Não & 1 & $(14,3)$ \\
\hline & & Local & 1 & $(14,3)$ \\
\hline & & Regional & 2 & $(28,6)$ \\
\hline & & Distância & 3 & $(42,8)$ \\
\hline \multirow[t]{4}{*}{ Locorregional } & 13 & Não & 8 & $(61,5)$ \\
\hline & & Local & 3 & $(23,1)$ \\
\hline & & Locorregional & 1 & $(7,7)$ \\
\hline & & Distância & 1 & $(7,7)$ \\
\hline
\end{tabular}

Dos pacientes que apresentaram segunda recidiva a distância, dois $(12,5 \%)$ foram submetidos à ressecção das metástases pulmonares e estavam vivos, sem doença, 10 anos e 12 anos após a ressecção. O tipo histológico de ambos era carcinoma adenocístico.

\subsection{Intervalo livre de doença}

Os pacientes que apresentaram recidiva antes de dois anos decorridos do tratamento inicial, a sobrevida em 5 anos foi $55 \%$. Por outro lado, os com recidivas tardia, (após dois anos) a sobrevida foi $80 \%$. Apesar 
de uma tendência a menor sobrevida nos pacientes com recidiva precoce, a diferença não é estatisticamente significante $(p=0,20)$ (Figura 11).

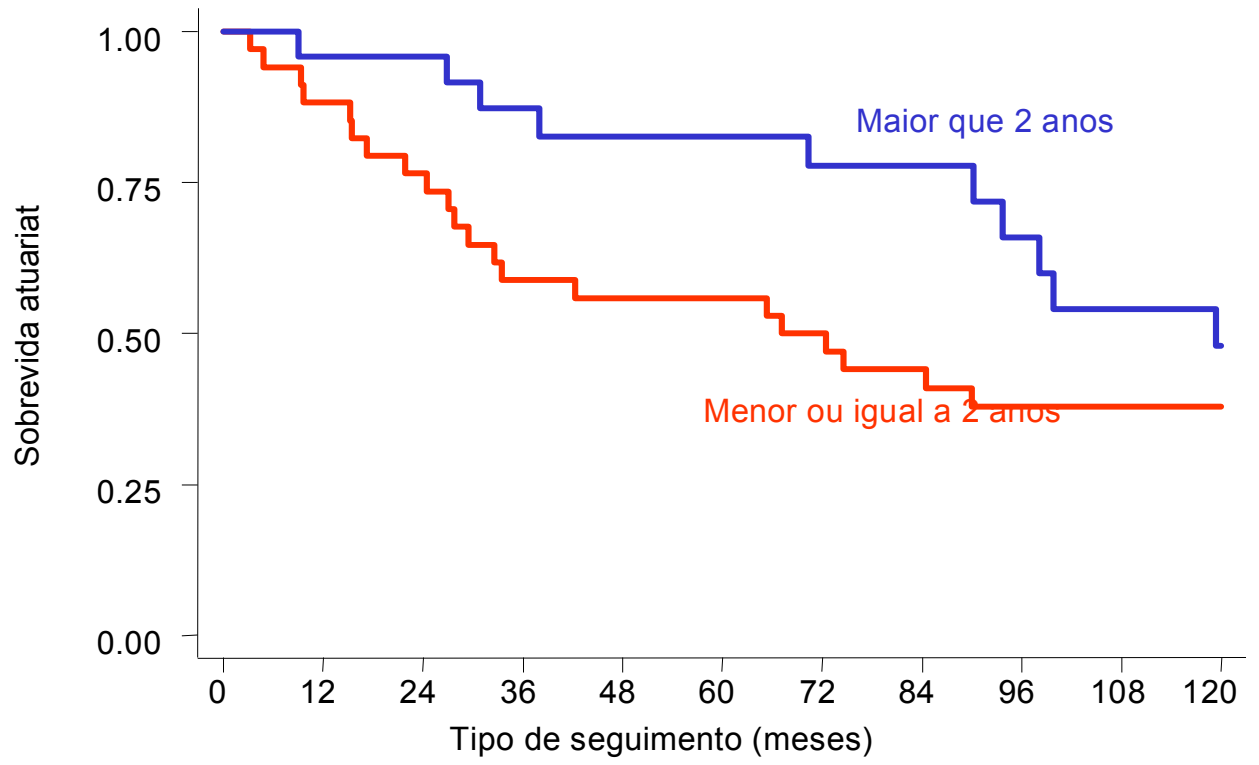

Figura 11. Curva de sobrevida atuarial de acordo com o tempo entre o tratamento inicial e a recorrência

\subsection{Variáveis demográficas}

\subsubsection{Faixa etária}

Foram adotadas conforme relatos da literatura (Poulsen et al. 1992, Bell et al. 2005) três faixas etárias: abaixo ou igual a 18 anos, de 19 a 60 anos e acima de 60 anos. A sobrevida global em 5 anos de pacientes com idade abaixo ou igual a 18 anos foi $71,4 \%$; nos pacientes com com idade entre 19 e 60 anos, foi $66,1 \%$, e nos pacientes com idade superior a 60 anos, foi $29,8 \%$. Estas diferenças foram significantes estatisticamente $(p=$ 0,001) (Tabela 5 e Figura 12) 


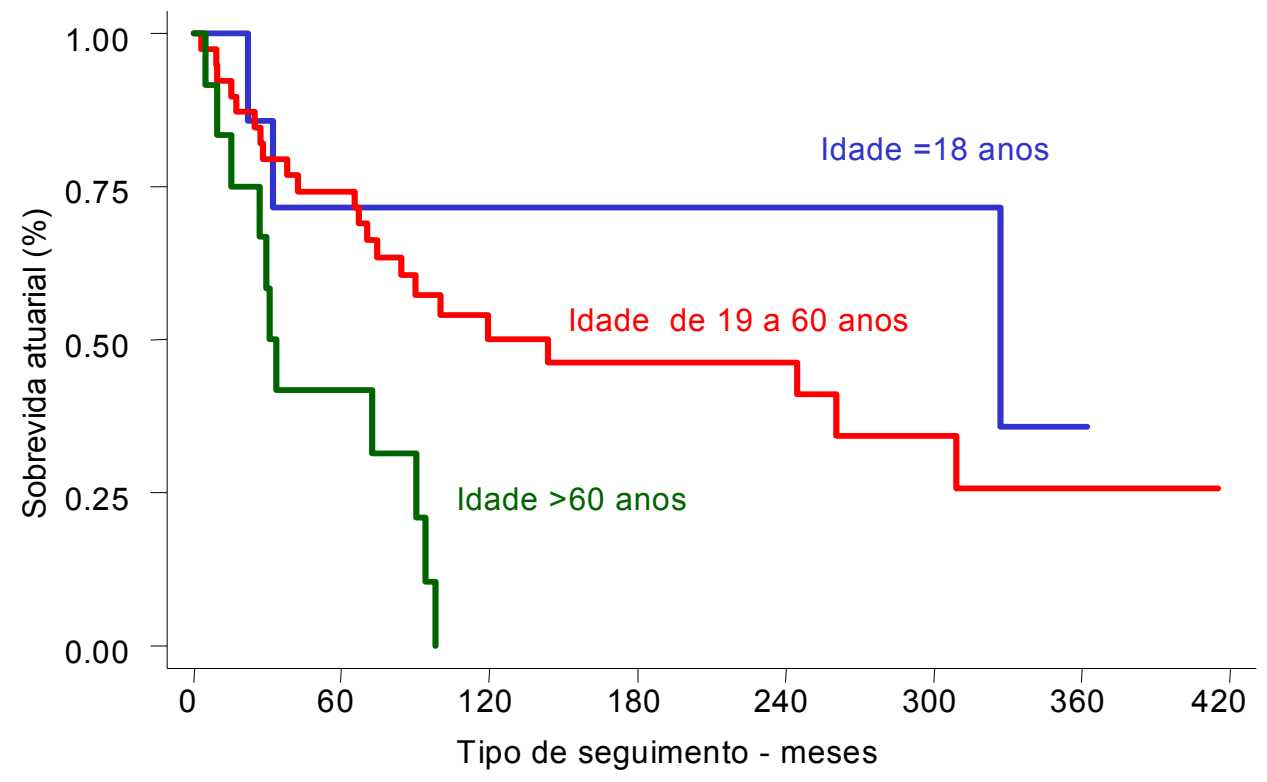

Figura 12. Curva de sobrevida atuarial global de acordo com a faixa etária.

\subsubsection{Sexo}

Houve predominância do sexo masculino e a relação entre os sexos masculino e feminino foi 1,4:1,0. A sobrevida global dos pacientes masculinos em 5 e 10 anos foi $70,1 \%$ e $47,6 \%$, respectivamente. Esta taxa de sobrevida foi melhor que a de pacientes do sexo feminino, porém não foi significativa $(p=0,39)($ Tabela 5$)$.

\subsubsection{Raça}

A sobrevida em 5 anos dos pacientes da raça branca foi $58,1 \%$ e daqueles de raça não branca, 66,7\%. Não houve diferença estatisticamente significante entre os grupos $(p=0,47)$ (Tabela 5$)$. 


\subsection{Variáveis clínicas}

\subsubsection{Localização do tumor primário}

A sobrevida dos portadores de carcinoma de parótida recidivados, em 5 e 10 anos foi 59,5 e 55,6\%, respectivamente. Nos portadores de tumores submandibulares foi 58,3 e $31,4 \%$. Nos pacientes com carcinoma originário de glândulas salivares menores da cavidade oral a sobrevida em 10 anos foi menor $(26,2 \%)$, porém esta diferença não foi significante $(p=0,34)($ Tabela $5)$.

\subsubsection{Local da recorrência}

Os pacientes que apresentaram recorrência local tiveram sobrevida em 5 e 10 anos de 70,1 e 47,2\%, respectivamente. Naqueles com recorrência locorregional, a sobrevida foi $37,8 \%$ em 5 anos. Pacientes com recorrência regional tiveram pior sobrevida em 5 anos 28,5\% em 10 anos, $14,3 \%$; entretanto, tais diferenças não foram estatisticamente significantes $(p=0,43)($ Tabela 5).

\subsubsection{Comprometimento ósseo e de pele}

$\mathrm{Na}$ avaliação clínica dos portadores de tumores recidivados, 15 (25,4\%) apresentaram comprometimento ósseo e $16 \quad(27,1 \%)$ comprometimento da pele. A sobrevida atuarial global em 5 anos dos pacientes sem e com comprometimento ósseo foi de $60,8 \%$ e $40,4 \%$, respectivamente. Esta diferença não foi estatisticamente signficante $(p=$ 
0,35). Nos pacientes sem e com comprometimento da pele a sobrevida em 5 anos foi $63,1 \%$ e $54,8 \%$, respectivamente. Tal diferença não foi estatisticamente significante $(p=0,58)$ (Tabela 5$)$.

\subsubsection{Estadiamento clínico da recidiva}

O estádio clínico da recorrência foi dividido em inicial, compreendendo os estádios clínicos I (rEC I) e II (rEC II) e avançado, agrupando os estádios clínicos III (rEC III) e IV (rECIV). A sobrevida de pacientes com tumores estádio clínico inicial, em 5 e 10 anos, foi 71,4 e 31,9\%, respectivamente. Nos casos com tumores em estádios avançados, a sobrevida dos pacientes em 5 e 10 anos foi 58,1 e 46,9\%. Não houve diferença significativa estatisticamente nas taxas de sobrevida dos pacientes com tumores estádios clínicos inicial e avançado $(p=0,63)$. (Tabela 5).

\subsubsection{Tipo histológico}

Adotou-se uma divisão dos tipos histológicos em baixo grau (carcinoma adenocístico e carcinoma de células acinares) e alto grau (carcinoma mucoepidermóide, adenocarcinoma, carcinoma epidermóide, carcinoma indiferenciado, carcinoma ex-adenoma pleomórfico). A sobrevida nos grupos de baixo grau e alto grau foi, em 5 anos, 52,5\% e 70,6\%, respectivamente. Não houve diferença significante entre os dois grupos de pacientes $(p=0,43)($ Tabela 5$)$. 


\subsubsection{Tipo de ressecção cirúrgica}

Os tipos de ressecção cirúrgica nos pacientes com tumores recidivados foram: ressecção do tumor primário, ressecção do tumor primário com esvaziamento cervical e esvaziamento cervical isoladamente. Trinta e nove pacientes $(66,1 \%)$, sofreram somente a ressecção do tumor primário com sobrevida em 5 e 10 anos de 66,7 e 33,3\%, respectivamente. Treze pacientes (22\%) tiveram ressecção do tumor e esvaziamento cervical, com sobrevida em 5 e 10 anos de $53,8 \%$ e 23,1\% respectivamente. Sete pacientes $(11,9 \%)$ foram submetidos somente a esvaziamento cervical, devido à recorrência regional. A sobrevida em 5 e 10 anos foi de 57,1 e $28,5 \%$, respectivamente. Não houve diferença estatisticamente significante entre os três grupos $(p=0,35)$ (Tabela 6).

\subsubsection{Conservação do nervo facial}

A conservação do nervo facial em tumores da parótida foi avaliada em 31 casos (52,5\%). O facial não foi conservado em 18 pacientes e em 13 houve sua preservação. A sobrevida em 5 anos de pacientes submetidos à cirurgia de resgate, na qual o nervo facial foi preservado foi de $66,4 \%$ e naqueles em que não houve tal preservação ela alcançou 47,9\%. Apesar de haver melhor sobrevida dos pacientes que preservaram o nervo facial em relação àqueles nos quais o nervo facial foi ressecado, esta diferença não foi significativa estatisticamente $(p=0,094)$ (Tabela 5). 


\subsubsection{Radioterapia adjuvante após a cirurgia de resgate}

A sobrevida de pacientes que realizaram radioterapia adjuvante foi em 5 e 10 anos atingiu 65,9 e 56,5\%, respectivamente. Para aqueles que não fizeram radioterapia, a sobrevida em 5 anos foi $57,9 \%$, esta diferença não foi significativa $(p=0,081)($ Tabela 6$)$.

Tabela 5. Sobrevida atuarial global após cirurgia de resgate de acordo com variáveis clínicas e anatomopatológicas.

\begin{tabular}{|c|c|c|c|c|}
\hline \multirow[b]{2}{*}{ Variáveis } & \multicolumn{4}{|c|}{ Sobrevida Global (\%) } \\
\hline & Categorias & 5-anos & 10-anos & p-valor \\
\hline $\begin{array}{l}\text { Faixa etária } \\
\text { (anos) }\end{array}$ & $\begin{array}{l}\leq 18 \\
19-60 \\
>60\end{array}$ & $\begin{array}{l}71,4 \\
66,1 \\
29,8\end{array}$ & $\begin{array}{l}71,4 \\
49,9 \\
0,00\end{array}$ & 0,001 \\
\hline Gênero & $\begin{array}{l}\text { Masculino } \\
\text { Feminino }\end{array}$ & $\begin{array}{l}70,1 \\
48,8\end{array}$ & $\begin{array}{l}47,6 \\
34,3\end{array}$ & 0,39 \\
\hline Topografia & $\begin{array}{l}\text { Parótida } \\
\text { Submandibular } \\
\text { Cavidade oral }\end{array}$ & $\begin{array}{l}59,5 \\
58,3 \\
69,2\end{array}$ & $\begin{array}{l}55,6 \\
31,4 \\
26,2\end{array}$ & 0,340 \\
\hline Recorrência & $\begin{array}{l}\text { Local } \\
\text { Regional } \\
\text { Locorregional }\end{array}$ & $\begin{array}{l}70,1 \\
28,5 \\
37,8\end{array}$ & $\begin{array}{l}47,2 \\
14,3 \\
37,8\end{array}$ & 0,437 \\
\hline $\begin{array}{l}\text { Estádio } \\
\text { Clínico }\end{array}$ & $\begin{array}{l}\text { Inicial } \\
\text { Avançado }\end{array}$ & $\begin{array}{l}71,4 \\
58,1\end{array}$ & $\begin{array}{l}31,9 \\
46,9\end{array}$ & 0,630 \\
\hline Pele positiva & $\begin{array}{l}\text { Não } \\
\text { Sim }\end{array}$ & $\begin{array}{l}63,7 \\
55,1\end{array}$ & $\begin{array}{l}39,7 \\
41,3\end{array}$ & 0,570 \\
\hline Osso positivo & $\begin{array}{l}\text { Não } \\
\text { Sim }\end{array}$ & $\begin{array}{l}62,3 \\
58,0\end{array}$ & $\begin{array}{l}43,1 \\
39,3\end{array}$ & 0,610 \\
\hline
\end{tabular}


Tabela 6. Sobrevida atuarial global após cirurgia de resgate de acordo com variáveis anatomopatológicas e terapêuticas.

\begin{tabular}{llccc}
\hline \multirow{2}{*}{$\begin{array}{l}\text { Variáveis } \\
\text { Anatomo }\end{array}$} & Categorias & 5 -anos & 10-anos & p-valor \\
patológico & Alto grau & 52,5 & 35,5 & 0,430 \\
& & 70,6 & 49,4 & \\
Tipo de & Primário & 66,7 & 33,3 & 0,351 \\
ressecção & Primário e EC & 53,8 & 23,1 & \\
cirúrgica & EC & 57,1 & 28,5 & \\
& & & & \\
Preservação & Não & 47,9 & 47,9 & 0,094 \\
do nervo facial & Sim & 66,4 & 66,4 & \\
& & & & \\
Radioterapia & Não & 57,9 & 29,8 & 0,081 \\
Adjuvante & Sim & 65,9 & 56,5 & \\
\hline
\end{tabular}




\section{DISCUSSÃO}

O princípio fundamental que deve nortear a escolha do melhor método de tratamento é a erradicação da doença com a máxima preservação da função do órgão, porém sem colocar em risco a radicalidade do procedimento terapêutico (Urken e Biller 1988). O tratamento pode ser considerado inadequado quando menos radical que o necessário para o controle da doença; por outro lado, pode ser excessivamente radical, impondo ao pacientes complicações e seqüelas desnecessárias.

A avaliação adequada da ressecabilidade desses tumores recorrentes, por meio do exame físico e de exames de imagem como tomografia computadorizada, além de uma avaliação clínica pré-operatória criteriosa são os pré-requisitos para a indicação precisa e bem fundamentada dessas cirurgias. O melhor conhecimento da resposta endócrino-metabólica ao trauma aplicado ao cuidado pós-operatório, o desenvolvimento das unidades de terapia intensiva e as novas técnicas anestésicas reduziram a morbi-mortalidade operatória, possibilitando cirurgias em pacientes que, no passado, apresentavam risco cirúrgico proibitivo (Kowalski 2000).

Poucos trabalhos na literatura abordam o tratamento da recorrência e não encontramos nenhum com desenho especifico para estudar a cirurgia de resgate em tumores malignos de glândulas salivares

A cirurgia de resgate é uma modalidade de tratamento para carcinoma epidermóide de boca recidivados. Agra et al. (2006) analisaram 
246 portadores de carcinomas de boca e orofaringe recidivados tratados por cirurgia de resgate. A sobrevida global em 5 anos foi de $32 \%$ e os fatores preditivos foram o estádio inicial (rEC I e rEC II) e o intervalo da recorrência após o tratamento inicial maior que um ano. Agra et al. (2003) ainda estudaram complicações advindas das cirurgias em monobloco de carcinomas de boca e orofaringe recidivados tratados com cirurgia de resgate. Sessenta e seis pacientes $(53,2 \%)$ apresentaram complicações, menores (infecção da ferida operatória, fístula e outras) em 42,7\% e maiores (ruptura de grande vaso e outras) em 18,5\%. A mortalidade operatória atingiu $3,2 \%$ dos pacientes.

As complicações após a cirurgia de resgate, em nossa série, ocorreram em $32,2 \%$ dos pacientes, sendo locais em $30,5 \%$ e sistêmicas em $1,7 \%$. As complicações locais mais freqüentes foram infecção da ferida operatória $(8,5 \%)$ e paralisia facial $(6,7 \%)$. Três pacientes apresentaram duas complicações locais: infecção operatória com necrose de retalhos ou fístula. Não ocorreram complicações maiores como a ruptura de grandes vasos ou perda completa do retalho. Nenhum paciente morreu no pósoperatório.

Parson et al. (1996) avaliaram portadores de glândulas salivares menores. Quarenta e quatro pacientes foram tratados inicialmente por cirurgia. Houve complicações pós-operatórias em seis pacientes (14\%). Dois pacientes tiveram complicações maiores (ruptura da carótida externa e necrose da língua). Os demais tiveram complicações menores: dois apresentaram infecção da ferida operatória, um fístula faringocutânea e 
outro perdeu $20 \%$ do retalho, necessitando de desbridamento. Dos 5 pacientes submetidos à cirurgia de resgate, um morreu no pós-operatório. Mendenhall et al. (2005) avaliaram 160 pacientes tratados por cirurgia e radioterapia adjuvante, dos quais $5(3,1 \%)$ apresentaram complicações pósoperatórias graves (um: necrose da língua; dois: hemorragia necessitando de reoperação; um apresentou fístula orocutânea precisando de fechamento por cirurgia e, outro, fratura de mandíbula. A mortalidade operatória foi $0,6 \%$ (um paciente após cirurgia craniofacial que evoluiu com meningite).

A sobrevida global dos portadores de carcinoma de glândulas salivares, não tratados previamente é variável, conforme o estudo. Em 5 anos variou de 51 até $81 \%$ e em 10 anos de 42 até 65\% (Spiro et al. 1975, Spiro et al. 1991, Regis de Brito Santos et al. 2001, Luukkaa et al. 2005, Therkildsen et al. 1998, Garden et al. 1994, Pedersen et al. 1992). North et al. (1990) reportaram taxas de sobrevida, em 5 e 10 anos, de 67 e 41\%, respectivamente. Este fato se deve ao perfil das populações estudadas pelos autores: como percentual de tumores de alto e baixo graus, proporção de carcinoma de glândulas salivares menores e maiores, topografia em seios da face (com pior prognóstico), entre outras. A sobrevida após tratamento da recorrência foi citada por Terhaard et al. (2004), que observou 85 pacientes com doença residual ou recorrência local/locorregional, submetidos a tratamento de resgate, sem especificar o tipo de tratamento, se cirurgia e/ou radioterapia. Em 5 anos, 32 pacientes (38\%) estavam vivos. Parsons et al. (1996) avaliaram cinco pacientes submetidos à cirurgia de resgate e somente um paciente teve a doença controlada, mas não há 
citação do tempo de seguimento. Tran et al. (1986) encontraram recorrência local em 13 pacientes, submetidos a tratamento de resgate (cirurgia, cirurgia com radioterapia pós-operatória, ou radioterapia isoladamente). Todos os pacientes que foram tratados somente com radioterapia morreram. Três pacientes (23\%) estavam vivos em 5 anos. Kagan et al. (1976) estudaram tumores recidivados da glândula parótida tratados com cirurgia e radioterapia. O seguimento médio foi curto, somente 2 anos. Dos cinqüenta e seis pacientes foram avaliados, 33 morreram da doença (59\%) e 23 estavam vivos, porém nove com doença em atividade. Spiro et al. (1982) estudaram 95 pacientes que apresentaram recorrência local e foram tratados (não há informação sobre o tipo de tratamento). Somente 12 pacientes $(12,6 \%)$ tiveram resposta. Não há informação sobre o tempo de seguimento. A sobrevida, após o tratamento da recorrência, variou nos estudos disponíveis na literatura, de 12 a 38\% em 5 anos.

Em nosso estudo, a sobrevida global em 5 anos foi $61 \%$ e em 10 anos $42 \%$, muito semelhante à encontrada por North et al. (1990). Este resultado é melhor que a sobrevida dos portadores de carcinoma epidermóide recidivado de boca e orofaringe tratados com cirurgia de resgate: $32 \%$ dos pacientes vivos em 5 anos (Agra et al. 2006). Também é compatível com a sobrevida relatada nos estudos que abordam o primeiro tratamento dos pacientes com carcinoma de glândulas salivares (Ribeiro et al. 2002, Regis de Brito Santos et al. 2001) e é superior aos resultados de resgates variados (Akbari et al. 2004, Hockel et al. 2006). É plausível considerar que em meio à casuística existe um importante viés pela indicação cirúrgica em tumores 
ressecáveis, em pacientes com poucas comorbidades e situação clínica favorável à indicação do tratamento.

O desenho dos estudos da literatura não focava o tratamento cirúrgico de resgate com intenção curativa. Em nosso estudo, houve melhor avaliação pré-operatória, quanto à ressecabilidade da lesão e das condições clínicas do paciente para uma cirurgia radical. Todos os pacientes selecionados não apresentaram doença residual após a cirurgia.

A sobrevida livre de doença é um fator prognóstico em algumas neoplasias recorrentes, como carcinoma epidermóide de cavidade oral e orofaringe. Llewelyn et al. (1997) estudaram 58 pacientes submetidos à cirurgia de resgate para carcinoma epidermóide de cavidade oral e encontraram uma associação direta entre o intervalo livre de doença e a sobrevida; houve melhora no prognóstico à medida que havia um aumento no intervalo livre de doença. Agra et al. (2006) avaliaram 246 pacientes com carcinoma epidermóide de boca e orofaringe, tratados por cirurgia de resgate. $\mathrm{O}$ intervalo livre de doença maior que um ano foi fator de melhor prognóstico na análise multivariada $(p=0,023)$. A recorrência precoce pode surgir devido à biologia desfavorável da neoplasia ou ao tratamento prévio inadequado. Infelizmente, os trabalhos sobre glândulas salivares não abordam a sobrevida livre de doença, como fator prognóstico em tumores malignos. Em nosso estudo, os pacientes com intervalo livre de doença curto, menor que dois anos, tiveram uma tendência a menor sobrevida em relação aos pacientes com recidiva acima de dois anos, mas esta diferença não foi significativa estatisticamente $(p=0,20)$. 
A idade tem sido considerada, em alguns estudos, como fator prognóstico em carcinomas de glândulas salivares não tratados previamente. Da Cruz Peres et al. (2005) também descreveram a idade (> 45 anos) como fator de pior prognóstico nos pacientes portadores de carcinoma adenocístico. Poulsen et al. (1992), analisando pacientes portadores de carcinoma de parótida tratados por cirurgia e radioterapia adjuvante, mostraram a idade acima de 60 anos como fator associado a um mau prognóstico. Kirkbride et al. (2001) também relatou que a idade acima de 55 anos é fator de mau prognóstico em relação à recorrência locorregional e sobrevida livre de doença.

A idade, em nossa série, considerando pacientes com mais de 60 anos, foi um fator de mau prognóstico na análise univariada $(p=0,001)$. Nove pacientes $(75 \%)$ apresentaram nova recidiva e todos morreram com a doença em atividade; $17 \%$ morreram de causas não relacionadas ao tumor e somente um paciente (8\%) estava vivo, sem doença, após 3 anos da cirurgia de resgate.

O sexo foi considerado fator prognóstico em alguns trabalhos, analisando pacientes sem tratamento prévio. Pires et al. (2004) e North et al. (1990) encontraram pior prognóstico em homens $(p=0,005)$ e $(p=0,008)$, respectivamente. Spiro et al. (1989) chegou ao mesmo resultado e sugeriu que este poderia decorrer de um maior proporção, em pacientes femininas, de tumores em estádio inicial e com baixo grau histológico (células acinares e carcinoma mucoepidermóide de baixo grau). Não há trabalhos com descrição adequada do sexo, em pacientes com recorrência. Em nosso 
estudo não houve diferença estatisticamente significante entre os sexos $(p=0,39)$, apesar de haver maior proporção de homens com tumores de células acinares $(80 \%)$.

A localização anatômica do tumor primário em pacientes sem tratamento prévio não é fator prognóstico independente em vários estudos (North et al. 1990, Bell et al. 2005, Luukkaa et al. 2005, Spiro et al. 1991), porém Spiro et al. (1989) encontraram, em análise multivariada, pior prognóstico em pacientes com tumores da glândula submandibular ( $p=$ 0,0007). Não há trabalhos com correlação adequada do prognóstico e localização do tumor primário em pacientes com recorrência. No presente estudo não foi encontrada diferença estatisticamente significante quanto à localização $(p=0,34)$.

O estadiamento clínico é um dos principais fatores preditivos, em tumores malignos de glândulas salivares não tratados previamente (Luukkaa et al. 2005, Regis de Brito Santos et al. 2001, Spiro 1998). Nos trabalhos sobre tumores malignos de glândulas salivares, abordando tratamento das recorrências não encontramos descrição adequada do estadiamento clínico dos pacientes. Em carcinoma epidermóide de boca recidivado, o estadiamento clínico da recorrência foi um importante fator prognóstico na análise multivariada (Agra et al. 2006). Em nosso estudo não houve diferença significativa na sobrevida dos pacientes com estádios clínicos inicial e avançado $(p=0,63)$. O estádio avançado ( $r E C$ III e rEC IV) compreendia $75 \%$ dos casos, sendo $60 \%$ rEC IV. 
O comprometimento ósseo e de pele foi avaliado por Terhaard et al. (2004) em portadores de tumores de glândulas salivares não tratados previamente. O comprometimento patológico ósseo e da pele foi fator de pior prognóstico na análise multivariada $(p=0,01$ e $p=0,04$, respectivamente). A invasão clínica da pele também foi fator prognóstico $(p=0,008)$. North et al. (1990) observaram que os pacientes com invasão da pele tiveram pior sobrevida na análise multivariada $(p=0,01)$. Não encontramos estudos com a adequada avaliação de margem óssea e de pele em pacientes com tumores recidivados de glândulas salivares. Em nosso estudo, a invasão clínica do osso e da pele não teve significância.

A preservação do nervo facial em pacientes com carcinoma de parótida tem sido reportada como fator prognóstico após tratamento inicial, quanto ao risco de recorrência local, em pacientes não tratados previamente. North et al. (1990) e Terhaard et al. (2004) verificaram que a paralisia facial estava relacionada a maior risco de recorrência local, porém sem redução da sobrevida. Em nosso estudo há uma tendência a melhor sobrevida de portadores de carcinoma de parótida tratados pela cirurgia de resgate, na qual o nervo facial foi preservado. Entretanto, este resultado não foi estatisticamente significante $(p=0,09)$. Ele está associado à extensão ou ao grau de agressividade tumoral, que possibilitaram a ressecção com margens satisfatórias.

A divisão dos pacientes quanto ao tipo histológico e grau de malignidade, varia conforme o estudo, dificultando análises comparativas. Leverstein et al. (1998) estudaram pacientes não tratados previamente e 
mostraram que o carcinoma de células acinares tem melhor prognóstico. Alguns estudos sugerem que o carcinoma de células acinares e o carcinoma mucoepidermóide de baixo grau possam ser agrupados em um grupo de baixo grau (Spiro 1986 e Spiro et al. 1989). Therkildsen et al. (1998) encontraram sobrevida melhor, no grupo de baixo grau (portadores de carcinoma de células acinares e carcinoma mucoepidermóide de baixo grau) $(p=0,01)$. North et al. (1990) mostraram que pacientes com carcinoma indiferenciado têm pior sobrevida na análise multivariada $(p=0,002)$. Yu et al. (1987) acharam que pacientes portadores de carcinoma de células acinares e carcinoma mucoepidermóide têm melhor prognóstico. Não encontramos na literatura estudos que avaliaram o tipo histológico nos pacientes com carcinomas recidivados de glândulas salivares. Em nosso estudo não houve diferença estatisticamente significante entre os grupos de alto e baixo grau, quanto à sobrevida global $(p=0,43)$.

É sempre difícil avaliar os diferentes tratamentos em um estudo retrospectivo; isto é particularmente verdadeiro em pacientes que receberam radioterapia pós-operatória. Diversos estudos avaliaram portadores de tumores de glândulas salivares não tratados previamente, submetidos a tratamento cirúrgico com e sem radioterapia adjuvante, porém não encontraram melhora na sobrevida (Terhaard et al. 2004 e 2005, Therkildsen et al. 1998, Spiro et al. 1991 e Spiro 1998), apenas melhora no controle locorregional (Therkildsen et al. 1998, Terhaard et al. 2004). Por outro lado, North et al. (1990) estudaram pacientes com carcinoma de glândulas salivares maiores e encontraram melhora da sobrevida em pacientes 
tratados com radioterapia adjuvante $(p=0,014)$. Armstrong et al. (1990) estudaram 46 portadores de carcinoma de glândulas salivares sem tratamento prévio tratados apenas com cirurgia, que foram pareados com 46 pacientes tratados com cirurgia e radioterapia. Houve melhora da sobrevida estatisticamente significante no grupo tratado com cirurgia e radioterapia pós-operatória. Em nossa série houve tendência à melhora da sobrevida em pacientes submetidos à cirurgia de resgate com radioterapia adjuvante, porém este resultado não foi significativo estatisticamente $(p=0,081)$. Não houve melhora do controle locorregional, provavelmente por causa das características dos pacientes irradiados (tumores mais avançados, nódulos positivos e outras).

A recorrência após a cirurgia de resgate não foi um tema especificamente abordado nos trabalhos disponíveis. Em nosso estudo, tal recorrência foi principalmente a distância, 16 casos (44\% das recidivas), sendo o tipo histológico mais freqüente o carcinoma adenocístico (68\%). Nos pacientes que apresentaram recorrência, somente 5 estão vivos após 10 anos dois com doença em atividade e três sem evidência de doença. Terhaard et al. (2004) encontraram sobrevida em pacientes com metástases de carcinoma adenocístico, de $30 \%$ em 5 anos. Nessa série, dois pacientes com recorrência a distância (pulmão), submetidos à ressecção pulmonar de resgate, estavam vivos após nove anos, sem evidência de doença. Em tumor quimio e radiorresistente (Locati et al. 2005 ), a ressecção cirúrgica de metástases pulmonares de carcinoma adenocístico pode ser realizada em casos selecionados. 
A identificação dos fatores relacionados ao prognóstico torna possível a melhor compreensão da evolução da doença, sendo de grande utilidade para a realização do tratamento mais adequado. A análise dos fatores prognósticos é uma arma essencial para definir subgrupos de pacientes com melhor ou pior evolução. Uma cuidadosa ponderação deve ser feita acerca dos resultados de todos os fatores envolvidos, porém se deve considerar que o controle adequado das variáveis quase sempre só pode ser obtido mediante estudos prospectivos aleatórios. Esses estudos, no caso de tumores malignos das glândulas salivares, tornam-se de difícil execução, dada sua baixa incidência na população. A identificação do perfil molecular desses tumores pode ter grande relevância prática e estudos futuros devem ser feitos para pesquisa desses marcadores moleculares. 


\section{CONCLUSÕES}

1. As cirurgias de resgate em pacientes com carcinomas recidivados de glândulas salivares podem ser realizadas com morbidade aceitável.

2. As complicações pós-operatórias foram predominantemente locais.

3. Somente a variável idade (> 60 anos) mostrou-se significantemente associada à piora do prognóstico. As demais variáveis demográficas, clínicas e terapêuticas não tiveram significância estatística no prognóstico. 


\section{ANEXO}

Cirurgia de resgate em tumores malignos de glândulas salivares recidivados
1) $\mathrm{RGH}$ $[\quad[\quad] \quad[\quad]-[]$
2) Idade $[\quad]$
3) Sexo (1) masc (2)fem
4) Raça (1)branco (2)negro (9)out
5) Topografia_(1)parótida (2)submandibular (3)cavidade oral (4)cav. NasaL (5) seio maxilar (6)seio etmóide (7)orofaringe (8)outros (9)ign.

[ ]

6) Estadiamento $T$ (2)T2 (3)T3 (4)T4 $(9) \lg n$

7) Estadiamento N_(1)No__(2)N1 (3) N2a $(4) \mathrm{N} 2 \mathrm{~b}$ (5)N2c (6)N3 (9) $\operatorname{lgn}$ [

8) Estadiamento clínico_(1)I (2)II (3)III (4)IV

9) Comprometimento da pele_(0)não

(1) $\operatorname{sim}$ (9)ign

10) Comprometimento ósseo_(0)não_(1)sim (9)ign

11) Comprometimento nervo__(0)não (1) $\operatorname{sim}$ (9)ign

12) Data da cirurgia

13) Tipo de cirurgia (1)PPCF (2)PTCF (3)PT (4)PTA (5)submaxilarec. (6)Res. Maxilar__(7)Res. Bucal (8) Maxilectomia (9)outro 
14) Esvaziamento cervical_(0)não (1)ESOH

(2)EC (3)ECB (9)ign [

15) AP__ (1)CA mucoepidermoide_(2)CA adenocistico (3)adenocarcinoma (4)CEC (5)CA indiferenciado (6)CA cel acinares (7)CA ex adenoma pleomorfico (9)outros

16) Embolização

perineural (0)não

(1) $\operatorname{sim}$

(9)ign

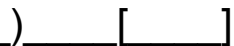

17) Embolização vascular (0)não_(1) $\operatorname{sim}$ (9)ign

18) Embolização linfática (0)não (1) sim (9)ign

19) Invasão óssea patológica (0)não_(1)sim (9)ign

20) Invasão pele patológica (0)não (1) $\operatorname{sim}$ (9)ign (0)não (1)sim_(_

21) Linfonodos comprometidos (9)ign

22) Linfonodos comprometidos contralateral (0)não (1)sim_(_ (9)ign

23) $N^{0} A P$ primário (9)ign $\left[{ }_{[}\right]$

24) Seqüência do tratamento (0)não (1) RXT (2) QT

25) Data da recorrência [ 1

26) Local da recorrência (1)local (2)pescoço homo (nível (3)pescoço contra (nível (4)locorregional [

27) Diagnóstico da recidiva (1)rotina 2)antes (3)depois (9)ign [ $]$

28) Sintomas (0)não (1) $\operatorname{sim}($ )

29) Fixação (0)não (1) $\operatorname{sim}$ 
30) Comprometimento da pele

(0)não

(1) sim

31) Comprometimento ósseo

(0)não

(1) sim

32) $R X$

(0)não

(1) $\operatorname{sim}$

33) US

(0)não

(1) sim

34) CT

(0)não

(1) sim

35) RNM

(0)não

(1) $\operatorname{sim}$

36) Diâmetro da recidiva

$\mathrm{cm}$ [

37) Biopsia da recidiva (0)não (1) $\operatorname{sim}$ (2)congelação

38) Resultado da BX (0)Sem biopsia (1)AP inconclusivo (2)AP - CA (3)congelação inconclusiva (4)Congelação CA

39) Estadiamento Tr_(1)T1 (2)T2 (3)T3

(4)T4 (9) $\lg n$

40) Estadiamento Nr_(1)NO (2)N1 (3) $\mathrm{N} 2 \mathrm{a}$ (4) N2b (5)N2c

(6)N3 (9) $\lg n$

41) Estadiamento clínico (1)! (2)II (3)III (4)IV

42) Ressecção da recidiva (1)PTCF (2)PT (3)PTA (4)ressecção ampliada região submandibular__(5)boca sem mandíbula (6) boca com mandíbula (7) maxilectomia (8)craniofacial

(9) orofaringe (10)laringectomia (11)outros (_) [

43) Radicalidade (1)sim (2)tumor residual

44) Conservação do n. facial (0)não (1) sim (9)não aplicável

45) Esvaziamento cervical homo (0)não (1)ESOH (2)EJC (3)EC radical (4)EC radical modificado (5)radical ampliado (9)ign 
46) Esvaziamento cervical contra (0)não

(1) $\mathrm{ESOH}$

(2)EJC

(3)EC radical

(4)EC radical modificado

(5)radical ampliado

(9)ign

47) $N^{\circ}$ AP recidiva

48) Reconstrução

(1)fechamento primário

(2)TL

(3)RR local

(4)peitoral (5)Outro miocutaneo

(6)microcirurgico

49) Complicações pós-resgate (locais)

(0)não

(1)infec FO

(2)paralisia facial

(3)seroma

(4)hematoma

(5)necrose de retalhos

(6)ruptura de grande vaso__(7)síndrome frey

(8)fístula (9)outra( )

[_ $[\quad]$

50) Complicações pós-resgate (sistêmicas)

(0)não_(1)BCP (2)ACV

(3)IAM (4)TVP

(5)TEP (6)Óbito pós-operatório (9)outra(

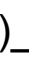
[

51) Tratamento pos resgate RXT (0)não

(1) Acel. Linear ( 2)cobalto (3)nêutron (4)conformacional

52) Tratamento pós resgaste QT_(0)não (1)curativa_(2)paliativa

53) Re-recidiva (0)não (1) $\operatorname{sim}$

54) Data da re-recorrência 1

55) Local da rerecorrência (1)local (2)pescoço homo (nível (3)pescoço contra (nível (4) locorregional (5)distância

56) Tratamento da re-recorrência (0)não (1)CIR (2)RXT (3)QT (4)RHD (5)outro 
57) Data da última informação 1

58) Situação nesta data (1)vivo s/ doença (2)vivo c/ doença (3)morte por CA (4)morte operatória (5)morte assintomática (6)P. de vista [ $]$ 
REFERÊNCIAS BIBLIOGRÁFICAS 


\section{REFERÊNCIAS BIBLIOGRÁFICAS}

Agra IM, Carvalho AL, Pontes E, Campos OD, Ulbrich FS, Magrin J, Kowalski LP. Postoperative complications after en bloc salvage surgery for head and neck cancer. Arch Otolaryngol Head Neck Surg. 2003;129(12):1317-21.

Agra IM, Carvalho AL, Ulbrich FS, de Campos OD, Martins EP, Magrin J Kowalski LP. Prognostic factors in salvage surgery for recurrent oral and oropharyngeal cancer. Head Neck. 2006;28(2):107-113.

Airoldi M, Pedani F, Brando V, Gabriele P, Giordano C. Cisplatin, epirubicin and 5-fluorouracil combination chemotherapy for recurrent carcinoma of the salivary gland. Tumori. 1989;75(3):252-6.

Airoldi M, Fornari G, Pedani F, Marchionatti S, Gabriele P, Succo G, Bumma C. Paclitaxel and carboplatin for recurrent salivary gland malignancies. Anticancer Res. 2000;20(5C):3781-3.

Airoldi M, Pedani F, Succo G, Gabriele AM, Ragona R, Marchionatti S, Bumma C. Phase II randomized trial comparing vinorelbine versus vinorelbine plus cisplatin in patients with recurrent salivary gland malignancies. Cancer. 2001;91(3):541-7.

Airoldi M, Cortesina G, Giordano C, Pedani F, Cavalot A, Marcato P, Beatrice $\mathrm{F}$, Bumma C. Update and perspectives on non-surgical treatment of salivary gland malignancies. Acta Otorhinolaryngol Ital. 2003;23(5):368-76.

Akbari RP, Paty PB, Guillem JG, Weiser MR, Temple LK, Minsky BD, Saltz L, Wong WD. Oncologic outcomes of salvage surgery for epidermoid carcinoma of the anus initially managed with combined modality therapy. Dis Colon Rectum. 2004;47(7):1136-44. 
Alcedo JC, Fabrega JM, Arosemena JR, Urrutia A. Imatinib mesylate as treatment for adenoid cystic carcinoma of the salivaryglands: report of two successfully treated cases. Head Neck. 2004;26(9):829-31.

Alves FA, Pires FR, de Almeida OP, Lopes MA, Kowalski LP. PCNA, Ki-67 and p53 expressions in submandibular salivary gland tumours. Int $\mathrm{J}$ Oral Maxillofac Surg. 2004;33(6):593-7.

Armstrong JG, Harrison LB, Spiro RH, Fass DE, Strong EW, Fuks ZY. Malignant tumors of major salivary gland origin. A matched-pair analysis of the role of combined surgery and postoperative radiotherapy. Arch Otolaryngol Head Neck Surg. 1990;116(3):290-3.

Armstrong JG, Harrison LB, Thaler HT, Friedlander-Klar $H$, Fass DE, Zelefsky MJ, Shah JP, Strong EW, Spiro RH. The indications for elective treatment of the neck in cancer of the major salivary glands. Cancer. 1992;69(3):615-9.

Bell RB, Dierks EJ, Homer L, Potter BE. Management and outcome of patients with malignant salivary gland tumors. J Oral Maxillofac Surg. 2005;63(7):917-28.

da Cruz Perez DE, Pires FR, Alves FA, Almeida OP, Kowalski LP. Salivary gland tumors in children and adolescents: a clinicopathologic and immunohistochemical study of fifty-three cases. Int $J$ Pediatr Otorhinolaryngol. 2004;68(7):895-902.

da Cruz Perez DE, de Abreu Alves F, Nobuko Nishimoto I, de Almeida OP, Kowalski LP. Prognostic factors in head and neck adenoid cystic carcinoma. Oral Oncol. 2006;42(2):139-46. 
Day TA, Deveikis J, Gillespie MB, Joe JK, Ogretmen B, Osguthorpe JD, Reed SG, Richardson MS, Rossi M, Saini R, Sharma AK, Stuart RK. Salivary gland neoplasms. Curr Treat Options Oncol. 2004;5(1):11-26.

Douglas JG, Laramore GE, Austin-Seymour M, Koh WJ, Lindsley KL, Cho P, Griffin TW. Neutron radiotherapy for adenoid cystic carcinoma of minor salivary glands. Int J Radiat Oncol Biol Phys. 1996;36(1):87-93.

Dreyfuss AI, Clark JR, Fallon BG, Posner MR, Norris CM Jr, Miller D. Cyclophosphamide, doxorubicin, and cisplatin combination chemotherapy for advanced carcinomas of salivary gland origin. Cancer. 1987;60(12):2869-72.

Duncan W, Orr JA, Arnott SJ, Jack WJ. Neutron therapy for malignant tumours of the salivary glands: a report of the Edinburgh experience. Radiother Oncol. 1987;8(2):97-104.

Etges A, Pinto DS Jr, Kowalski LP, Soares FA, Araujo VC. Salivary duct carcinoma: immunohistochemical profile of an aggressive salivary gland tumour. J Clin Pathol. 2003;56(12):914-8.

Ferlito A, Pellitteri PK, Robbins KT, Shaha AR, Kowalski LP, Silver CE, Anniko M, Rinaldo A, Medina JE, Bradley PJ, Byers RM. Management of the neck in cancer of the major salivary glands, thyroid and parathyroid glands.Acta Otolaryngol. 2002;122(6):673-8.

Fiorella R, Di Nicola V, Fiorella ML, Spinelli DA, Coppola F, Luperto P, Madami L. Major salivary gland diseases. multicentre study. Acta Otorhinolaryngol Ital. 2005;25(3):182-90. Erratum in: Acta Otorhinolaryngol Ital. 2005;25(5):following 337.

Fitzpatrick PJ, Theriault C. Malignant salivary gland tumors. Int $J$ Radiat Oncol Biol Phys. 1986;12(10):1743-7. 
Gallo O, Franchi A, Bianchi S, Boddi V, Giannelli E, Alajmo E. p53 oncoprotein expression in parotid gland carcinoma is associated with clinical outcome. Cancer. 1995;75(8):2037-44.

Garden AS, Weber RS, Ang KK, Morrison WH, Matre J, Peters LJ. Postoperative radiation therapy for malignant tumors of minor salivary glands. Outcome and patterns of failure. Cancer. 1994;73(10):2563-9.

Gilbert J, Li Y, Pinto HA, Jennings T, Kies MS, Silverman P, Forastiere AA. Phase II trial of taxol in salivary gland malignancies (E1394): a trial of the Eastern Cooperative Oncology Group. Head Neck. 2006;28(3):197-204.

Griffin TW, Pajak TF, Laramore GE, Duncan W, Richter MP, Hendrickson FR, Maor MH. Neutron vs photon irradiation of inoperable salivary gland tumors: results of an RTOG-MRC Cooperative Randomized Study. Int $J$ Radiat Oncol Biol Phys. 1988;15(5):1085-90.

Harish K. Management of primary malignant epithelial parotid tumors. Surg Oncol. 2004;13(1):7-16.

Harrison LB, Armstrong JG, Spiro RH, Fass DE, Strong EW. Postoperative radiation therapy for major salivary gland malignancies. $J$ Surg Oncol. 1990;45(1):52-5.

Hockel M. Ultra-radical compartmentalized surgery in gynaecological oncology. Eur J Surg Oncol. 2006;32(8):859-65.

Hocwald E, Korkmaz H, Yoo GH, Adsay V, Shibuya TY, Abrams J, Jacobs JR. Prognostic factors in major salivary gland cancer. Laryngoscope. 2001;111(8):1434-9. 
Holst VA, Marshall CE, Moskaluk CA, Frierson HF Jr. KIT protein expression and analysis of c-kit gene mutation in adenoid cystic carcinoma. Mod Pathol. 1999;12(10):956-60.

Hotte SJ, Winquist EW, Lamont E, MacKenzie M, Vokes E, Chen EX, Brown S, Pond GR, Murgo A, Siu LL. Imatinib mesylate in patients with adenoid cystic cancers of the salivary glands expressing c-kit: a Princess Margaret Hospital phase II consortium study. J Clin Oncol. 2005;23(3):585-90.

Huber PE, Debus J, Latz D, Zierhut D, Bischof M, Wannenmacher M, Engenhart-Cabillic R. Radiotherapy for advanced adenoid cystic carcinoma: neutrons, photons or mixed beam? Radiother Oncol. 2001;59(2):161-7.

Jansisyanont P, Blanchaert RH Jr, Ord RA. Intraoral minor salivary gland neoplasm: a single institution experience of 80 cases. Int $\mathrm{J}$ Oral Maxillofac Surg. 2002;31(3):257-61.

Jeng YM, Lin CY, Hsu HC. Expression of the c-kit protein is associated with certain subtypes of salivary gland carcinoma. Cancer Lett. 2000;154(1):10711.

Kagan AR, Nussbaum H, Handler S, Shapiro R, Gilbert HA, Jacobs M, Miles JW, Chan PY, Calcaterra T. Recurrences from malignant parotid salivary gland tumors. Cancer. 1976;37(6):2600-4.

Kirkbride P, Liu FF, O'Sullivan B, Payne D, Warde P, Gullane P, Pintilie M, Keane $\mathrm{TJ}$, Cummings B. Outcome of curative management of malignant tumours of the parotid gland. J Otolaryngol. 2001;30(5):271-9. 
Kokemueller H, Swennen G, Brueggemann N, Brachvogel P, Eckardt A, Hausamen JE. Epithelial malignancies of the salivary glands: clinical experience of a single institution-a review. Int $J$ Oral Maxillofac Surg. 2004;33(5):423-32.

Kowalski LP. Strategies in the management in recurrent and inoperable cancer. In: Proceedings of the $4^{\circ}$ International Conference on Head Neck Cancer; 2000 Jul 28 Aug 4; San Francisco, USA.

Krause CJ. The management of parotid neoplasms. Head Neck Surg. 1981;3(4):340-3.

Laurie SA, Licitra L. Systemic therapy in the palliative management of advanced salivary gland cancers. J Clin Oncol. 2006;24(17):2673-8.

Leverstein $\mathrm{H}$, van der Wal JE, Tiwari RM, Tobi $\mathrm{H}$, van der Waal I, Mehta DM, Snow GB. Malignant epithelial parotid gland tumours: analysis and results in 65 previously untreated patients. Br J Surg. 1998;85(9):1267-72.

Licitra L, Cavina R, Grandi C, Palma SD, Guzzo M, Demicheli R, Molinari R. Cisplatin, doxorubicin and cyclophosphamide in advanced salivary gland carcinoma: a phase II trial of 22 patients. Ann Oncol. 1996;7(6):640-2.

Lima RA, Tavares MR, Dias FL, Kligerman J, Nascimento MF, Barbosa MM, Cernea CR, Soares JR, Santos IC, Salviano S. Clinical prognostic factors in malignant parotid gland tumors. Otolaryngol Head Neck Surg. 2005;133(5):702-8.

Llewelyn J, Mitchell R. Survival of patients who needed salvage surgery for recurrence after radiotherapy for oral carcinoma. $\mathrm{Br} J$ Oral Maxillofac Surg. 1997;35(6):424-18. 
Locati LD, Guzzo M, Bossi P, Massone PP, Conti B, Fumagalli E, Bareggi C, Cantu G, Licitra L. Lung metastasectomy in adenoid cystic carcinoma (ACC) of salivary gland. Oral Oncol. 2005;41(9):890-4.

Lopes MA, Santos GC, Kowalski LP. Multivariate survival analysis of 128 cases of oral cavity minor salivary gland carcinomas. Head Neck. 1998;20(8):699-706.

Luukkaa H, Klemi P, Leivo I, Koivunen P, Laranne J, Makitie A, Virtaniemi J, Hinkka S, Grenman R. Salivary gland cancer in Finland 1991--96: an evaluation of 237 cases. Acta Otolaryngol. 2005;125(2):207-14.

Medina JE. Neck dissection in the treatment of cancer of major salivary glands. Otolaryngol Clin North Am. 1998;31(5):815-22.

Mendenhall WM, Morris CG, Amdur RJ, Werning JW, Villaret DB. Radiotherapy alone or combined with surgery for salivary gland carcinoma. Cancer. 2005;103(12):2544-50.

North CA, Lee DJ, Piantadosi S, Zahurak M, Johns ME. Carcinoma of the major salivary glands treated by surgery or surgery plus postoperative radiotherapy. Int J Radiat Oncol Biol Phys. 1990;18(6):1319-26.

Parkin DM, Whelan SL, Ferlay J, et al. Cancer incidence in five continents. Lyon: International Agency for Research on Cancer; 1997. p.808-9. (Scientific Publication $\left.n^{\circ} 14\right)$.

Parsons JT, Mendenhall WM, Stringer SP, Cassisi NJ, Million RR. Management of minor salivary gland carcinomas. Int $J$ Radiat Oncol Biol Phys. 1996;35(3):443-54. 
Pedersen D, Overgaard J, Sogaard H, Elbrond O, Overgaard M. Malignant parotid tumors in 110 consecutive patients: treatment results and prognosis. Laryngoscope. 1992;102(9):1064-9.

Perez DE, Pires FR, Alves Fde A, Almeida OP, Kowalski LP. Sublingual salivary gland tumors: clinicopathologic study of six cases.Oral Surg Oral Med Oral Pathol Oral Radiol Endod. 2005;100(4):449-53.

Pires FR, de Almeida OP, de Araujo VC, Kowalski LP. Prognostic factors in head and neck mucoepidermoid carcinoma. Arch Otolaryngol Head Neck Surg. 2004;130(2):174-80.

Pohar S, Gay H, Rosenbaum P, Klish D, Bogart J, Sagerman R, Hsu J, Kellman R. Malignant parotid tumors: presentation, clinical/pathologic prognostic factors, and treatment outcomes. Int $J$ Radiat Oncol Biol Phys. 20051;61(1):112-8.

Poulsen MG, Pratt GR, Kynaston B, Tripcony LB. Prognostic variables in malignant epithelial tumors of the parotid. Int $J$ Radiat Oncol Biol Phys. 1992;23(2):327-32.

Regis De Brito Santos I, Kowalski LP, Cavalcante De Araujo V, Flavia Logullo A, Magrin J. Multivariate analysis of risk factors for neck metastases in surgically treated parotid carcinomas. Arch Otolaryngol Head Neck Surg. 2001;127(1):56-60.

Renehan A, Gleave EN, Hancock BD, Smith P, McGurk M. Long-term follow-up of over 1000 patients with salivary gland tumours treated in a single centre. Br J Surg. 1996;83(12):1750-4. 
Ribeiro Kde C, Kowalski LP, Saba LM, de Camargo B. Epithelial salivary glands neoplasms in children and adolescents: a forty-four-year experience. Med Pediatr Oncol. 2002;39(6):594-600.

Rinaldo A, Ferlito A, Pellitteri PK, Robbins KT, Shaha AR, Bradley PJ, Kowalski LP, Wei WI. Management of malignant submandibular gland tumors. Acta Otolaryngol. 2003;123(8):896-904.

Saroja KR, Hendrickson FR, Cohen L, Mansell J, Lennox A. Re-irradiation of locally recurrent tumors with fast neutrons. Int $J$ Radiat Oncol Biol Phys. 1988;15(1):115-21.

Satko I, Stanko P, Longauerova I. Salivary gland tumours treated in the stomatological clinics in Bratislava. J Craniomaxillofac Surg. 2000;28(1):5661.

Seifert G, Sobin LH. The World Health Organization's Histological Classification of Salivary Gland Tumors: a commentary on the second edition. Cancer. 1992;70(2):379-85.

Speight PM, Barrett AW. Salivary gland tumours. Oral Dis. 2002;8(5):229-40.

Spiro RH, Huvos AG, Strong EW. Cancer of the parotid gland: a clinicopathologic study of 288 primary cases. Am J Surg. 1975 Oct;130(4):452-9.

Spiro RH, Huvos AG, Strong EW. Adenocarcinoma of salivary origin: clinicopathologic study of 204 patients. Am J Surg. 1982;144(4):423-31.

Spiro RH. Salivary neoplasms: overview of a 35-year experience with 2,807 patients. Head Neck Surg. 1986;8(3):177-84. 
Spiro RH, Armstrong J, Harrison L, Geller NL, Lin SY, Strong EW. Carcinoma of major salivary glands. Recent trends. Arch Otolaryngol Head Neck Surg. 1989;115(3):316-321.

Spiro RH, Thaler HT, Hicks WF, Kher UA, Huvos AH, Strong EW. The importance of clinical staging of minor salivary gland carcinoma. Am J Surg. 1991;162(4):330-6.

Spiro RH. Management of malignant tumors of the salivary glands. Oncology (Williston Park). 1998;12(5):671-80; discussion 683.

Suen JY, Johns ME. Chemotherapy for salivary gland cancer. Laryngoscope. 1982;92(3):235-9.

Terhaard $\mathrm{CH}$, Lubsen $\mathrm{H}$, Van der Tweel I, Hilgers FJ, Eijkenboom WM, Marres HA, Tjho-Heslinga RE, de Jong JM, Roodenburg JL; Dutch Head and Neck Oncology Cooperative Group. Salivary gland carcinoma: independent prognostic factors for locorregional control, distant metastases, and overall survival: results of the Dutch head and neck oncology cooperative group. Head Neck. 2004;26(8):681-692; discussion 692-693.

Terhaard $\mathrm{CH}$, Lubsen $\mathrm{H}$, Rasch CR, Levendag PC, Kaanders HH, TjhoHeslinga RE, van Den Ende PL, Burlage F; Dutch Head and Neck Oncology Cooperative Group. The role of radiotherapy in the treatment of malignant salivary gland tumors. Int J Radiat Oncol Biol Phys. 2005;61(1):103-11.

Therkildsen MH, Christensen M, Andersen LJ, Schiodt T, Hansen HS. Salivary gland carcinomas: prognostic factors. Acta Oncol. 1998;37(7-8):70113.

Trackray AC, Sobin LH.Histological typing of salivary gland tumours. World Health Organization. International histological classification of tumours. 1972 
Tran L, Sadeghi A, Hanson D, Juillard G, Mackintosh R, Calcaterra TC, Parker RG. Major salivary gland tumors: treatment results and prognostic factors. Laryngoscope. 1986;96(10):1139-44.

Yu GY, Ma DQ. Carcinoma of the salivary gland: a clinicopathologic study of 405 cases. Semin Surg Oncol. 1987;3(4):240-4.

Urken ML, Biller HF. Management of early vocal cord carcinoma. Oncology (Williston Park). 1988;2(4):48-62.

Vered M, Braunstein E, Buchner A. Immunohistochemical study of epidermal growth factor receptor in adenoid cystic carcinoma of salivary gland origin. Head Neck. 2002;24(7):632-6.

Wahlberg P, Anderson H, Biorklund A, Moller T, Perfekt R. Carcinoma of the parotid and submandibular glands--a study of survival in 2465 patients. Oral Oncol. 2002;38(7):706-13.

Wang CC, Goodman M. Photon irradiation of unresectable carcinomas of salivary glands. Int J Radiat Oncol Biol Phys. 1991;21(3):569-76.

Zbaren P, Schupbach J, Nuyens M, Stauffer E, Greiner R, Hausler R. Carcinoma of the parotid gland. Am J Surg. 2003;186(1):57-62. 